Journal of

Nutrigenetics

Nutrigenomics
J Nutrigenet Nutrigenomics 2016;9:151-210

\title{
Abstracts
}

\section{0th Congress of the International Society of Nutrigenetics/Nutrigenomics (ISNN)}

May 22-26, 2016, Tel Aviv, Israel

\section{Guest Editors}

Zohar Kerem, Rehovot

J. Alfredo Martínez, Navarra

Ram Reifen, Rehovot

\section{Contents}

\section{Oral Presentations \\ Abstracts 01-014 \\ 166 Poster Presentations \\ Abstracts P15-P53 \\ 205 Selected Abstracts \\ Abstracts 54-57}




\section{Oral Presentations}

\section{1}

'Junk Food' Diet Inhibits Longitudinal Growth and Bone Quality through Impairment of Growth Plate Development in Young Rodents

Janna Zaretsky, Shelley Griess-Fishheimer, Tamara Travinsky, Sveta Penn, Efrat Monsonego-Ornan

Institute of Biochemistry and Nutrition, The Hebrew University, Israel

In recent decades there has been a radical change in the western society food and beverage consumption habits alongside with sedentary lifestyle. Junk foods and soft drinks are high in fat and refined sugars while not providing the appropriate levels of macro and micro-nutrients for growth requirements of children. The purpose of our studies was to establish a junk food western diet model in rodents, in order to examine its influence on skeletal development. Our main focus was the process of endochondral ossification taking place in the growth plates. An in-vivo experiment was conducted on post weaning rodents representing the growth period in human until sexual maturation and growthplates closure. These studies demonstrated that junk food consumption in young age resulted in longitudinal growth and weight gain retardation. Furthermore, disruption of bone quality in the cortical and the trabecular areas was observed, such as a reduction in BMD, BV/TV, cortical thickness and load to fracture. This phenomenon occurred in conjunction with a radical growth plate phenotype characterized by a plaque of avascular non calcified cartilage in the metaphysis. Based on these findings, we suggest that the consumption of unbalanced junk food diet in addition to its known undesirable impact on metabolic state in young age, also leads to a direct damage to bone and cartilage cells, impairing post-natal longitudinal growth. 


\title{
02
}

\section{Colostrum, and Its Oxytocin, Attenuate ER-Stress and Inflammation Signaling Markers, in Rat Newborn Gut Villi}

\author{
Benjamin Klein ${ }^{1}$, Hadassah Tamir ${ }^{2,3}$, Martha Welch ${ }^{1,3}$ \\ ${ }^{1}$ Department of Developmental Neuroscience, New York State Psychiatric \\ Institute, Columbia University College of Physicians and Surgeons, USA; \\ ${ }^{2}$ Division of Molecular Imaging and Neuropathology, New York State \\ Psychiatric Institute, Columbia University College of Physicians and \\ Surgeons, USA; ${ }^{3}$ Department of Pathology \& Cell Biology, Columbia \\ University College of Physicians and Surgeons, USA
}

Naïve newborn-type enterocytes of gut villi form a thin barrier between inner tissues and the outer gut luminal microbiota and nutritional elements during postnatal development. Rat newborn enterocytes overexpress oxytocin (OT) receptor (OTR) until weaning, this implicates milk OT in gut development. We found, in adult enterocytes cell-line, that OT induces ER stress response by increasing the BiP/GRP78 chaperone and by down regulation of mRNA translation via phosphorylation of translation initiation factor 2a (eIF2a). Bacterial endotoxin (LPS) antagonized this response. To test the in-vivo impact of milk on ER stress response we compared (by immuno-capillary electrophoresis) BiP/ GRP78 and phospho-eIF2a (p-eIF2a) abundance between villi unprimed and those primed by colostrum. Unprimed villi showed high BiP/GRP78 while colostrum decreased it in primed villi. p-eIF2a, detected in unprimed villi coordinately with high BiP/GRP78, grew higher in primed villi, contrary to expected p-eIF2a and BjP/GRP78 coordinate decrease upon ER stress relief. Cytoplasmic dsRNA-induced eIF2a kinase (PKR) was phosphorylated (pPKR) hence active in primed versus unprimed villi, suggesting that colostrum inactivated eIF2a via pPKR. Colostrum has also strongly increased IkB, the active inhibitor of the inflammatory transcription factor (NF-kB). Exogenously added OT, significantly increased IkB. Unprimed cultured villi incubated with colostrum in the presence of OTR antagonist showed that BiP, IkB, and autophagosome initiation marker LC3A, (that responds to ER stress) are all partially dependent on OT contained in colostrum. These results indicate that colostrum assisted by its OT attenuates signaling of inflammation and the ER stress response markers during the critical period when villi development is finalized concomitantly with exposure to microbiota. Further intensification of p-eIF2 translation inhibition marker implicates OT, present in colostrum, in safeguarding newborn villi against overwhelming unnecessary translation activity of the mammary mRNA repertoire present in milk. Thus colostrum may protect villi ER from excessive stressful protein cargo. 


\title{
03
}

Maternal Diet Enrichment in Alpha Linolenic or Saturated Fatty Acids Differentially Regulates Gene Expression in Mice Offspring's Liver

\author{
Alicia Leikin Frenkel ${ }^{1,2}$, Limor Shomonov-Wagner ${ }^{1}$, Ana Juknat ${ }^{3,4}$, \\ Metsada Pasmanik-Chor ${ }^{5}$ \\ ${ }^{1}$ The Sackler Faculty of Medicine, Sheba, Tel Hashomer, Tel Aviv \\ University, Israel; ${ }^{2}$ Bert Strassburger Lipid Center, Sheba, Tel Hashomer, \\ Tel Aviv University, Israel; ${ }^{3}$ Adelson Center for the Biology of Addictive \\ Diseases, Sackler Faculty of Medicine, Tel Aviv University, Israel; \\ ${ }^{4}$ Neurobiology Department, Weizmann Institute of Science, Israel; \\ ${ }^{5}$ Bioinformatics Unit, G.S.W. Faculty of Life Sciences, Tel-Aviv University, \\ Israel
}

Background/Aims: Lipid metabolic disarray in young and adult mice offspring's liver is induced by saturated fatty acids (SFA) but prevented by alpha linolenic acid (ALA, 18:3 $\omega 3$ ) in the maternal diet during pregnancy and lactation. The aim of the present study was to analyse the impact of maternal dietary ALA on the liver gene expression in the new-born offspring in comparison to a SFA diet.

Methods: C57Bl6/J dams were fed with diets normal in calories but rich in ALA or SFA before mating and during pregnancy. Pups were sacrificed at birth and liver parameters were assessed. Gene expression was characterized by microarray analysis and validated by real time qPCR.

Results: ALA compared to SFA in maternal diets during pregnancy, increased polyunsaturated fatty acids while differentially modified fatty acid desaturase activities in offspring liver. Overall, 474 and 662 genes from born pups' liver, were differentially regulated by ALA and SFA compared to control diet $(\mathrm{P}=0.05$; Fold change 2), respectively. Notably, Per3 was up-regulated by ALA whereas down-regulated by SFA, compared to control diet.

Conclusions: ALA and SFA enriched diets differentially affect gene expression pattern in the offspring's liver. ALA in particular, upregulates genes associated to low adiposity. 


\section{4}

\section{Connecting the Dots: Bioengineering Legume Seeds with Enhanced Nutritional Composition Using Systems Biology Approaches}

Ziyue Huang, Chuan Jiang, Yuanyuan Zhang, Yimin She, Renyi Liu, Jerome Verdier

Shanghai Institute for Plant Stress Biology (PSC), Shanghai Institutes of Biological Sciences (SIBS), Chinese Academy of Sciences (CAS), China

Legume seeds are a primary source of proteins, lipids, carbohydrates, secondary metabolites, and minerals. Some regulatory genes have been discovered that control legume seed storage metabolism but the whole seed storage regulation is still unknown. Therefore, we have initiated a systems biology approach in combination with functional genomics to decipher seed storage metabolism in the model legume, Medicago truncatula. To unravel regulatory mechanisms, we have been using different approaches to model the seed development and create a virtual seed network (SeedMap). An in silico gene regulatory network during seed development was built, which linked transcripts, metabolites, proteins, hormones, minerals and small RNA. In parallel, a genomewide association study using resources developed by the Medicago HapMap project has been initiated and should reveal sequence polymorphisms associated with distinct seed composition phenotypes such as carbohydrate, protein, lipid, secondary metabolite and mineral contents. The combination of these approaches has been leading to the discovery of genes controlling seed nutritional composition and seed quality traits in legumes, which are currently functionally analysed. This approach is leading to new ways to improve the nutritional quality of legume seeds and some examples of how using these approaches to bioengineer seed with enhanced nutritional quality will be highlighted. 


\section{5}

\section{Securolida: Genomic Aspect of a Natural Antineoplastic New} Drug and Its Pharmaceutical Development

\section{Jose Terrero, Santo Domingo}

Universidad Nacional Evangelica, Dominican Republic

The pharmaceutical industry, in a sustained manner, for decades has been representing one of greater thrust business activities globally and the Dominican Republic is no exception. Although the growth of the Dominican pharmaceutical industry has been based in the development of drugs with active substances released from the intellectual property protection. The present work shows genomic aspects in the path you need to explore for the development of a drug, whose active ingredient is under own patent. Shows also the exercise of years of laboratory Magnachem, a visionary company that has managed to achieve an important number of the stages to develop BIOSEC, a new drug whose active ingredient is SECUROLIDA, a novel molecule that this laboratory has developed into the world of the antineoplastics. 


\section{6}

Anti-Aging Effect of Marine Fish Oil and Polyunsaturated Fatty Acids Based on Redox State Regulation and Telomere Protection Mechanisms

Jingnan Chen Yan Wei, Jun Wang, Jiahui Chen, Yan Zhang, Yu Zhang

College of Biosystems Engineering and Food Science, Zhejiang University, China

Introduction: To understand the anti-aging mechanism of polyunsaturated fatty acids (PUFA) and provide scientific basis for the fish-rich Mediterranean diet, we systematically investigated the effect of fish oil (FO), docosahexenoic acid (DHA) and arachidonic acid (AA) on the redox state regulation and chromosome telomere protection in D-galactose-induced aging mice after 8-week oral administration.

Results: (i) Antioxidase activities (superoxide dismutase, catalase, glutathione peroxidase) in liver, heart, brain and plasma were significantly improved by both FO and PUFA interventions $(P=0.05)$; (ii) Levels of plasma F2-isoprostane, and malonaldehyde (MDA) and the monoamine oxidase (MAO) activities in brain were reduced by $20 \%-79 \%, 16 \%-54 \%$ and $50 \%-90 \%$ in all experimental groups, respectively; (iii) However, PUFA monomers in high and moderate doses dramatically increased MDA levels in liver, while high and low 0 doses of AA even enhanced MDA levels in heart; (iv) $\omega-3$ rather than $\omega-6$ PUFA prevented liver against telomere shortening, while only FO protected chromosome telomere in testis $(P=0.05)$; (v) PUFA monomers exerted better inhibitory effect on the expression of oncogene c-Myc if compared with the effect of FO $(P=0.05)$. Both PUFA and FO could effectively inactivate p53 response to telomere dysfunction.

Conclusions: FO and PUFA monomers in low doses have positive in vivo redox state regulation effect. Only $\omega-3$ PUFA is capable of rescuing age-related telomere shortening. The anti-aging effect of $\omega$-3 PUFA appears dose-dependent while the effect of FO is also related to its PUFA composition especially the ratio of DHA/eicosapentaenoic acid (EPA). 


\section{7}

\section{Nutrigenomic Mechanisms Associated with Chemoprevention by Phytochemicals; A Clinical Perspective}

\section{Christine Houghton}

School of Human Movement and Nutrition Sciences, University of

Queensland, Australia

Epidemiological studies show that diets rich in fruits and vegetables are protective against cancer and chronic disease. However, attempts to translate these epidemiological findings into clinical practice have not yielded the anticipated benefits.

The chemoprotective benefits of plant foods have been broadly attributed to the presence of antioxidant vitamins but large-scale clinical trials using supplemental vitamins have been almost universally negative. Nevertheless, antioxidant supplements are popular amongst consumers, including those at risk of or with diagnosed cancer.

The emerging science of Nutrigenomics considers alternative mechanisms by which plant foods may modulate cellular defences. Plant-derived phytochemicals have been shown to influence signalling pathways within human cells. This presentation will highlight the chemoprotective effect of plant-derived bioactives, focusing on the transcription factor Nrf2, capable of influencing the expression of several hundred cytoprotective genes. Nrf2 is influenced by stressors which include phytochemicals, exercise and environmental toxins as activators of endogenous cellular defences.

Recent elucidation of signalling pathways is enabling the development of strategies to activate the cell's multifaceted endogenous defence mechanisms. Knowing how phytochemicals influence cellular defence systems may in time yield to more targeted therapies.

This discussion will examine biochemical pathways capable of activating endogenous cellular defences and in so doing will review several mechanisms by which cancer cells may be directly and indirectly targeted. Furthermore, it will describe some of the phytochemicals with the potential to activate such mechanisms, highlighting those derived from cruciferous vegetables and referring to estrogen metabolism as an example. Several commonly-occurring gene polymorphisms or SNPs are relevant to the discussion as is reference to bioavailability and the dose-response.

In searching for simple solutions to chemoprevention, the science of Nutrigenomics represents a clinical paradigm which would appear safe, effective, quantifiable and able to be readily implemented. Its potential long-term clinical benefit is yet to be fully evaluated. 


\section{8}

\section{High Dose Phytochemicals in Clinical Trials: Friend or Foe?}

Jeremy Johnson, Kelly Deyo

Yue-Ting Wang Pharmacy Practice, University of Illinois at Chicago, USA

A variety of health attributes have been attributed to resveratrol from dietary sources with epidemiology studies suggesting a possible role in reducing the risk and progression of many diseases including cancer, diabetes, and cardiovascular disease. Often these studies identify quantities of resveratrol less than $10 \mathrm{mg}$ per day as having health promoting properties. In contrast, the majority of clinical trials often utilize doses as high as 2.5 grams per day as a strategy to overcome the perception that resveratrol pharmacokinetics need to be optimized. Strategies to enhance absorption including various drug delivery vehicles or absorption enhancers have been utilized in animal models successfully to increases the systemic exposure of resveratrol. In our most recent study, human subjects diagnosed with GI neuroendocrine tumors were administered 2.5 grams of resveratrol daily for 84 days to understand the impact on long term administration of resveratrol on plasma levels 1 hour post administration. Plasma samples were analyzed by LC/MS for resveratrol and resveratrol-conjugates. Interestingly, plasma levels at days 56 and 84 were approximately $50 \%$ less than plasma levels obtained on day 28 . These results begin to question the perceived benefits of high dose administration of resveratrol on plasma pharmacokinetics over extended periods. 


\section{9}

\section{A Combination of Single-Nucleotide Polymorphisms Is Associated with Interindividual Variability in Dietary Beta-Carotene Bioavailability in Healthy Men}

Charles Desmarchelier Marion Nowicki, Romain Bott, Patrick Borel

Nutrition, Obesity and Risk of Thrombosis, UMR 1062 INSERM/1260

INRA/Aix-Marseille University, France

The bioavailability of beta-carotene $(\beta C)$, the main dietary provitamin A carotenoid, varies among individuals. It is not known whether this variability can affect long-term $\beta C$, and hence vitamin $\mathrm{A}$, status. We hypothesized that variations in genes involved in $\beta C$ absorption and postprandial metabolism could at least partially explain the high interindividual variability in $\beta C$ bioavailability. Thus, the main objectives of this study were to identify associated single-nucleotide polymorphisms (SNPs), and to estimate whether populations with different allele frequencies at these SNPs could have different abilities to absorb provitamin A carotenoids.

In this single-group design, 33 healthy, nonobese adult men were genotyped with the use of whole-genome microarrays. After an overnight fast, they consumed a test meal containing $100 \mathrm{~g}$ tomato puree providing $0.4 \mathrm{mg} \beta \mathrm{C}$. The postprandial plasma chylomicron $\beta C$ concentration was then measured at regular time intervals over $8 \mathrm{~h}$. Partial least squares (PLS) regression was used to identify the best combination of SNPs in or near candidate genes ( 54 genes representing 2172 SNPs) that was associated with the postprandial chylomicron $\beta C$ response (incremental $\beta C$ area-under-the-curve concentration over $8 \mathrm{~h}$ in chylomicrons).

The postprandial chylomicron $\beta C$ response was highly variable $(\mathrm{CV}=105 \%)$ and was positively correlated with the fasting plasma $\beta C$ concentration $(r=0.78$; $\mathrm{P}=0.001)$. A significant $\left(\mathrm{P}=6.54 \times 10^{-3}\right)$ multivalidated PLS regression model, which included 25 SNPs in 12 genes, explained $69 \%$ of the variance in the postprandial chylomicron $\beta C$ response, i.e. $\beta C$ bioavailability.

Interindividual variability in $\beta C$ bioavailability appears to be partially modulated by a combination of SNPs in 12 genes. This variability likely affects the long-term blood $\beta C$ status. A theoretic calculation of $\beta C$ bioavailability in 4 populations of the international HapMap I project suggests that populations with different allele frequencies in these SNPs might exhibit a different ability to absorb dietary $\beta$ C. 


\section{0}

\section{$\mathrm{N}$-Acetyl-L-Cysteine Administration in Early Life Affect the Development of Glucose Intolerance and Diabetes in Adulthood}

Alona Falach ${ }^{1,2}$, Hava Rozenfeld 1,2, Lital Argaev-Frenkel1, Sanford R. Sampson ${ }^{3}$, Tovit Rosenzweig ${ }^{1}$

${ }^{1}$ Departments of Molecular Biology and Nutritional Studies, Ariel University, Israel; ${ }^{2}$ Faculty of Life Sciences, Bar-Ilan University, Israel; ${ }^{3}$ Department of Molecular Cell Biology, Weizmann Institute of Science, Israel

Early life environment has consequences on health in adulthood. Oxidative stress has been suggested to be involved in fetal programming, leading to elevated risks for several metabolic disorders, including type 2 diabetes (T2D). However, the possibility that antioxidant consumption during early life may affect the development of diabetes has scarcely been studied.

In order to investigate this hypothesis, mice were treated with N-acetyl-Lcysteine (NAC) during pregnancy and suckling, and the manifestations of diabetes were followed in offspring. Two animal models were used in this study; KK-Ay mice, a genetic model of T2D, and high fat diet (HFD)-fed obese mice.

KK-Ay mice showed improvement in glucose and insulin tolerance. While fasting insulin was not affected, glucose-induced insulin secretion was lower in offspring of NAC-treated mice, suggesting improved sensitivity to the hormone. In addition, an increase in insulin-induced phosphorylation of AMPK and PKB was observed in liver but not in skeletal muscle. No difference was detected in pyruvate challenge test and mRNA level of gluconeogenic enzymes, suggesting that gluconeogenesis is not affected by the early exposure to NAC, however some improvement in the severity of hepatic steatosis was observed. Markers of oxidative stress in serum had not been affected by the treatment, suggesting that NAC is involved in mechanisms of metabolic programming rather than having a prolong effect on oxidative state.

In the HFD-fed mice body weight accumulation was lower in the offspring of NAC-treated mice, compared to untreated HFD-fed littermates. Beneficial effect of NAC consumption on glucose tolerance was also found in this model.

In conclusion, NAC consumption during early life improves glucose metabolism in adulthood. In order to recommend NAC consumption for the prevention of glucose intolerance and T2D, the mechanisms underlying the observed effects should be well characterized. 


\section{1 Differential Inhibition of Oxidative Stress by Specific Cereal Grain Fractions: In vitro and in vivo Studies \\ Kabo Masisi', Khuong Le ${ }^{3}$, William Diehl-Jones², Mohammed Moghadasian ${ }^{3}$, Trust Beta ${ }^{1}$ \\ ${ }^{1}$ Food Science and Richardson Centre for Functional Foods \& Nutraceuticals, University of Manitoba, Canada; ${ }^{2}$ Biological Science and Children's Hospital Research Institute of Manitoba, University of Manitoba, Canada; ${ }^{3}$ Human Nutritional Science and Canadian Centre for Agri-Food Research in Health \& Medicine, University of Manitoba, Canada}

The effects of hand-separated aleurone, endosperm and germ fractions of barley, corn and wheat on the cellular antioxidant activity and antioxidant pathway genes (84 genes related to oxidative stress) were determined. Moreover, antioxidant enzymes (superoxide dismutase (SOD), glutathione peroxidase (GPx) and catalase (CAT)), oxidative stress indicator (1,1-diphenyl-2-picrylhydrazyl-scavenging (DPPH)) in plasma and liver, and abundance of the antioxidant enzymes and oxidative stress-related genes in the liver of low density lipoprotein receptor knockout (LDL-r-KO) mice were assessed. In study 1 (in vitro), using 3-(4,5-dimethylthiazolyl-2)-2,5-diphenyltetrazolium bromide (MTT) assay, 2,2' azobis (2-amidinopropane) dihydrochloride (AAPH)-induced cell loss was effectively reduced by pre-incubating Caco-2, HT-29 and FHs 74 Int cells with carotenoid extracts from cereal grain fractions. Moreover, the extracts reduced $(\mathrm{P}=0.001) \mathrm{AAPH}$-induced intracellular oxidation in the cell lines, suggesting antioxidant activity. Of the 84 antioxidant pathway genes included in microarray array analysis (HT-29 cells), the expression of 28 genes were enhanced ( $\mathrm{P}=0.05)$. In study 2 (in vivo), four groups male LDL-r-KO mice ( $\mathrm{n}=$ 32; 8 mice/group) were fed with the experimental diets supplemented with (3 treated groups) or without (1 control group) $5 \%(\mathrm{w} / \mathrm{w}$ ) of each hand-separated corn fraction for 10 weeks. All diets were supplemented with $0.06 \%$ (w/w) dietary cholesterol. Consumption of corn fractions significantly increased antioxidant capacity as measured by DPPH $(\mathrm{P}=0.0003)$ and the activity of the CAT $(\mathrm{P}=0.01)$ and GPx $(\mathrm{P}=0.0001)$. The activity of SOD $(\mathrm{P}=0.01)$ was decreased as compared with the control mice. The abundance of CAT, GPx1 and SOD1 genes was not significantly affected by the treatments. Our findings suggest that cereal grain fractions improved oxidative status, and thus have the potential to mitigate oxidative stress. This study will have significant contribution to cellular and LDL oxidation and, will encourage further investigations of potential oxidative stress benefits of cereal grain fractions and further understanding of other mechanisms of action. 


\title{
012
}

\section{How Enrichment of Milk with Magnesium Can Improve} Quality and Safety of Milk Products?

\author{
Moshe Shemesh ${ }^{1}$, Hilla Oknin ${ }^{1,2}$, Doron Steinberg ${ }^{2}$, Yulia Gololobova ${ }^{1}$, \\ Solange Bernstein ${ }^{1}$ \\ ${ }^{1}$ Department of Food Quality and Safety, Institute for Postharvest \\ Technology and Food Sciences, Agricultural Research Organization (ARO), \\ The Volcani Center, Israel; ${ }^{2}$ Institute of Dental Sciences, Faculty of Dental \\ Medicine, Hebrew University-Hadassah, Israel
}

Magnesium is attracting increasing attention in the functional foods and supplements industry. Several clinical studies have highlighted the beneficial effect of this important mineral on human health. Here, we report that magnesium ions significantly inhibit biofilm formation by Bacillus species which represent a continuous hygiene problem and can lead to serious economic losses due to food spoilage. The inhibitory effect of magnesium ions within milk was notable even at $1 \mathrm{mM}$ concentration, while at $5 \mathrm{mM}$ of $\mathrm{MgCl} 2$ the biofilm bundles were almost totally diminished. Notably, bacterial growth was not significantly affected in a response to $\mathrm{Mg} 2+$ ions; hence, the mode of action of $\mathrm{Mg} 2+$ ions is apparently specific to inhibition of biofilm formation. Biofilm formation depends on the synthesis of extracellular matrix, whose production in $B$. subtilis is specified by two major operons: the epsA-O and tapA operons. We analyzed the effect of Mg2+ ions on matrix gene expression using transcriptional fusions of tapA promoter to the gene encoding cyan fluorescent protein (CFP). The expression of the tapA operon was reduced drastically in response to $\mathrm{Mg} 2+$ ions suggesting about their inhibitory effect on expression of the matrix genes in B. subtilis. Our further observations indicate about hypersensitivity of bacterial cells, in the presence of $\mathrm{Mg} 2+$, to some traditional treatments undertaken during milk processing. Taken together, we propose that milk products fortified with magnesium could be considered as healthier as well as safer food for human consumption. 


\section{3}

\section{Expression and Biological Function of miRNA in Breast Milk}

Regina Golan-Gerstl' ${ }^{1}$, Yaffa Elbaum Shiff', Shimon Reif ${ }^{1}$

${ }^{1}$ Department of Pediatrics, Hadassah Medical Center, Israel; ${ }^{2}$ The school of Nutritional Sciences, The Hebrew University, Israel

Introduction: Breastfeeding protects against neonatal necrotizing enterocolitis and other infant infections. Several epidemiological studies have demonstrated that breastfeeding can reduce the rate of childhood leukemia. Recent studies, in our lab and elsewhere, have demonstrated that mammalian milk contains miRNAs in the fat layer and skim milk.

We therefore, hypotheses that in milk from different mammalian sources are beneficial miRNAs that can be involved in the differentiation and development of intestine and in the preventive effect of milk in childhood diseases such as leukemia.

Methods: Milk miRNA were isolated from the two layers of milk. Next sequencing generation and Real Time PCR were performed to study the profile expression of miRNA in milk. Normal and tumor cells were incubated with exosomes and fat globules derived from milk and the profile expression of miRNA was analyzed by real time PCR.

Results: We have identified several known important miRNA in the skim and fat fraction of breast milk at different time of lactation. We demonstrated that miRNAs related to immune system development and diseases prevention such as mir-148a-3p and mir-146 have different levels of expression at different periods of lactation, as well between different mothers. In addition, breast milk exosomes and the miRNA from the lipid fraction are capable to enter into normal and cancer cells, transferring miRNAs into them and changing the miRNA profile of those cells.

Conclusion: We found expression of beneficial miRNA in milk that can regulate target genes with potential molecular and physiological functions, which might account for the observed immunity-based benefits those breast-fed infants. Our results will give the experimental basis in attempt to add beneficial miRNA from milk to infant formula. 


\section{4}

\section{Impact of Food Pesticides on the Gut Microbiota}

Rosita Gabbianelli ${ }^{1}$, Donatella Fedeli ${ }^{1}$, Laura Bordoni ${ }^{2}$, Dennis Fiorini ${ }^{3}$, Ivan Dus ${ }^{4}$, Cinzia Nasuti ${ }^{1}$

${ }^{1}$ School of Pharmacy, ${ }^{2}$ School of Advanced Studies, ${ }^{3}$ School of Biosciences and Veterinary Medicine, University of Camerino, Italy; ${ }^{4}$ Head of Research for Chanson Water Company, Taiwan

The presence of pyrethroid pesticides in food is largely documented by many studies, which underline that dietary intake represents one of the main font of pyrethroid toxicity in humans. Their use is related to the protection of crops against pests in order to guarantee the increased request of food for people over the world. The relevant presence of pyrethroid metabolites in the urine of population confirms their presence in food, demonstrating that it is a global problem.

Our previous studies demonstrated that exposure to permethrin, during early life, can modify Nurr1 gene expression and that the treatment with pesticide leads to a decrease of dopamine level in striatum of rats. The damage could be counterbalanced by the co-treatment with protective bioactive compounds. Here we tested the effect of electrolyzed reduced water (ERW), prepared by using a Chanson Revolution 9 plates (Taiwan), in rats treated in early life with permethrin. Gut microbiota analysis and short chain fatty acids were analyzed.

Results show a decreased Lachnospira strain in permethrin treated rats with respect to control ones, and the co-treatment with ERW increases this strain. Furthermore, an increase in Roseburia and Blautia was observed in co-treated rats. A positive effect of ERW was observed on fecal SCFA because butyric acid resulted significantly increased.

The employ of ERW as functional water could be useful for health to counterbalance the damage due to pesticide intake through the food. 


\title{
Poster Presentations
}

\section{P15}

\section{Myricetin Improves Endurance Capacity and Mitochondrial} Function by Activating SIRT1 and PGC-1a

\author{
Hoe-Yune Jung ${ }^{1,5}$, Hye-Guk Ryu ${ }^{1}$, Bo-Hwa Choi ${ }^{2}$, Dongyeop Lee ${ }^{1}$, \\ Younghoon $\mathrm{Go}^{3}$, Seung-Jae Lee ${ }^{1}$, In-Kyu Lee ${ }^{3}$, Sung Woo Jeong ${ }^{4}$, \\ Kyong Tai Kim ${ }^{1}$ \\ ${ }^{1}$ Department of Life Science, Division of Molecular and Life Science, \\ Pohang University of Science and Technology (POSTECH), South Korea; \\ ${ }^{2}$ Pohang Center for Evaluation of Biomaterials, Pohang Technopark, South \\ Korea; ${ }^{3}$ Department of Internal Medicine, Kyungpook National University \\ School of Medicine, South Korea; ${ }^{4}$ Green-Bio Materials Research TFT, \\ Research Institute of Industrial Science \& Technology, South Korea; ${ }^{5}$ \&\&D \\ Center, NovMetaPharma Co., Ltd., South Korea
}

The beneficial effects of exercise on overall health make it desirable to identify the orally active agents that enhance the effects of exercise in an effort to cure metabolic diseases. Diminished mitochondrial oxidative phosphorylation and aerobic capacity are associated with reduced longevity. We tested whether myricetin from many vegetables such as paprika, which is known to extend lifespan in c. elegans, impacts mitochondrial function. Treatment of mice with myricetin significantly increased their aerobic capacity, as evidenced by their increased running time and consumption of oxygen. And also, treatment of $c$. elegans with myricetin significantly improved healthspan and lifespan. In the mouse, morphometric analysis of the various tissues mitochondria, by electron microscopy, revealed clearly larger mitochondrial structures. This amplification of the mitochondria was reflected both in the quantification of mitochondrial size and mitochondrial mtDNA content and also improves citrate synthase enzyme activity. Myricetin's effects were associated with an induction of genes for oxidative phosphorylation and mitochondrial biogenesis and were largely explained by an myricetin-mediated decrease in PGC-1a acetylation and an increase in Sirt1 activity. Additionally, Liver and kidney histology was similar between the myricetin-treated and phosphate-buffered saline-treated animals, indicating that KME was nontoxic. Taken together, our data show that myricetin induces mitochondrial activity, possibly by activating PGC-1a and SIRT1, and improves the endurance of mice, strongly suggesting that myricetin has great potential as a novel mitochondria-activating agent. 


\title{
P16
}

\section{Angiotensin-Converting Enzyme (ACE) I/D Polymorphism Influences Body Composition through the Hydration Status in a Young Italian Population}

\author{
Laura Bordoni ${ }^{1}$, Francesca Marchegiani ${ }^{2}$, Valerio Napolioni ${ }^{3}$, \\ Rosita Gabbianelli ${ }^{4}$ \\ ${ }^{1}$ School of Advanced Studies, University of Camerino, Camerino, Italy; \\ ${ }^{2}$ Clinical Laboratory and Molecular Diagnostics, INRCA-IRCCS, Ancona, \\ Italy; ${ }^{3}$ Department of Neurology and Neurological Sciences, Stanford \\ University, School of Medicine, Stanford, CA, USA; ${ }^{4}$ School of Pharmacy, \\ University of Camerino, Camerino, Italy
}

Objective: The well-known insertion/deletion polymorphism (rs4646994) of the human angiotensin-converting enzyme (ACE) gene has been previously associated with obesity both in preschoolers, adolescents and adults. It has been reported a consistent association between this polymorphism and blood flow, muscular strength and ACE enzyme activity. Despite the relevant role of ACE in all these processes, very few evidences are currently available on the relationship between this gene variant and hydration status. Thus, we studied the association between ACE I/D polymorphism, body composition and hydration status in a young population of Italians children and adolescents.

Methods: A total of 306 healthy children and adolescents, who regularly practice sport, were recruited for the study. For all the subjects, anthropometric, bioimpedentiometric parameters and urine samples were collected. ACE I/D genotyping was performed on DNA from buccal swabs. General linear model (GLM) and two-way ANOVA tests has been performed with SPSS software.

Results: On the whole, interesting correlation between ACE I/D polymorphism, body composition and hydration status was detected in our population. In particular, a genetic-dependent effect of a good hydration status on body composition has been identified.

Conclusion: Our results do not only confirm the relationship between ACE I/D polymorphism and body composition, but also suggest a key role of the hydration status on the modulation of this relationship. These interesting preliminary results warrant further investigation to disentangle the genetic role of ACE on hydration homeostasis. 


\section{P17 \\ Effects of Dietary Supplementation with Eicosapentaenoic Acid and/or $\alpha$-Lipoic Acid on Adipose Tissue Transcriptome in Healthy Overweight/Obese Women}

Ana E. Huerta ${ }^{1,2}$, Pedro L. Prieto-Hontoria ${ }^{1,5}$, Fernández-Galilea Marta ${ }^{1,6}$, J. Alfredo Martínez $^{1-4}$, María J. Moreno-Aliaga ${ }^{1-4}$

${ }^{1}$ Department of Nutrition, Food Science and Physiology, University of Navarra, Pamplona, Spain; ${ }^{2}$ Centre for Nutrition Research, University of Navarra, Pamplona, Spain; ${ }^{3}$ CIBER Fisiopatologia de la Obesidad y Nutricion (CIBERobn), Instituto de Salud Carlos III (ISCIII), Madrid, Spain;

${ }^{4}$ Navarra Institute for Health Research, (IdiSNA), Pamplona, Spain; ${ }^{5}$ Current Affiliation: Faculty of Health and Physical Activity Science, University SEK, Chile; ${ }^{6}$ Current Affiliation: Department of Nutrition, Diabetes and Metabolism, School of Medicine, Pontificia Universidad Catolica de Chile, Santiago de Chile, Chile

Introduction: The important role of white adipose tissue on obesity-related clinical complications has been widely described. In the present study we aimed to characterize the transcriptomic changes promoted by supplementation with eicosapentaenoic acid (EPA) and/or $\alpha$-lipoic acid in adipose tissue of overweight/obese healthy women following an energy-restricted diet, using microarrays.

Methods: Healthy overweight/obese women underwent an energyrestricted diet (30\% of total energy expenditure) during 10 weeks in four parallel intervention groups, which varied only in the product supplementation [Control, EPA (1.3 g/d), a-lipoic acid (0.3 g/d), or EPA+a-lipoic acid (1.3 g/d + 0.3 g/d)]. Subcutaneous abdominal adipose tissue biopsies were obtained at the end of the intervention. A total of 33297 genes were analyzed using the Affymetrix Human Gene 1.1 ST 24-Array plate.

Results: The bioinformatics analysis identified 580 genes with differential expression ( $\mathrm{P}=0.05$ ) between groups. Also, 72 genes (27 downregulated and 45 upregulated) were commonly differentiated in the three supplemented groups. Furthermore, a total of 120 genes (48 downregulated and 72 upregulated) were differentially expressed in both groups supplemented with $\alpha$-lipoic acid; while a total of 83 genes ( 26 downregulated and 57 upregulated) were differential in the groups supplemented with EPA. Furthermore, the KEGG analysis revealed that the lysosome and genes related insulin signaling pathways were significantly regulated in the groups supplemented with EPA (EPA and EPA+ $\alpha$-lipoic acid). Moreover in the group supplemented with both compounds, the cell adhesion molecule processes were significantly influenced. Interestingly, the Ingenuity Pathway Analysis revealed a differential expression in lipid metabolism and inflammation pathways in the groups supplemented with $\alpha$-lipoic acid, while in the group supplemented with EPA, biological functions related with migration and adhesion of cells were modified.

Conclusions: Supplementation with a-lipoic acid and/or EPA induce transcriptomic changes in adipose tissue, which could account for the metabolic effects observed for these bioactive food components. 


\section{P18}

\section{Prediction of Obesity Risk through Three Genetic Risk Score (GRS) Models}

Leticia Goni ${ }^{1,2}$, Marta Cuervo ${ }^{1-4}$, Fermín I. Milagro ${ }^{1-3}$, J. Alfredo Martínez ${ }^{1-4}$

${ }^{1}$ Department of Nutrition, Food Science and Physiology, University of Navarra, Spain; ${ }^{2}$ Centre for Nutrition Research, University of Navarra, Spain; ${ }^{3}$ CIBER Fisiopatologia Obesidad y Nutricion (CIBERobn), Instituto de Salud Carlos III, Spain; ${ }^{4}$ Navarra Institute for Health Research, IdiSNA, Spain

Introduction: Different genetic risk score (GRSs) models are being developed to assess the risk of obesity. The main aim of this study was to determine the best model of GRS to predict the risk of obesity in a Spanish population and to investigate the effect of age.

Methods: A total of 708 Caucasians, were examined for anthropometric characteristics, body composition, food habits and physical activity. Oral epithelial cells were collected for the genotyping of three polymorphisms (rs9939609, rs17782313 and rs1801282) located in or near FTO, MC4R and PPARG genes, respectively. GRSs were calculated by three different methods previously described: (1) simple count of allele risk, (2) weighted GRS based on published ORs and (3) weighted GRS based on published $\beta$ s.

Results: Of the total population, $41.8 \%(n=296)$ were obese. The unadjusted logistic regression model indicated an odds ratio for obesity of 1.17 ( $\mathrm{p}=$ 0.039), 1.13 ( $\mathrm{p}=0.034$ ) and 1.85 ( $\mathrm{p}=0.013$ ) for GRS1, GRS2 and GRS3, respectively. After adjusted for covariates (age, sex, energy intake and physical activity) only GRS 3 showed a significant association (OR 2.00, $\mathrm{p}=0.010$ ). When the fitstat analysis was applied, the Bayesian Information Criterion (BIC) analysis showed a positive support for the GRS 3 model, both unadjusted and adjusted for covariates. The association of GRS 3 with obesity was also analysed according to three groups of age (18-30 y.o., 31-45 y.o. and 46-84 y.o.). After adjusting for covariates, the Area Under the Curve (AUC) was $0.83,0.71$ and 0.69 for young adults, adults and old adults, respectively.

Conclusions: A weighted GRS based on published $\beta s$ is better than a simple count of risk alleles GRS or a weighted GRS based on published ORs for the prediction of obesity risk. Moreover, these results indicate that GRS 3 fits better in young adults than in older people, suggesting that the obesity prediction declines with age. 


\title{
P19 \\ DNA Methylation Levels of miR-1203, miR-412 and miR-216A Coding Sequences in White Blood Cells Are Associated with Childhood Obesity
}

\author{
Marcos Garcia-Lacarte ${ }^{1,2}$, María Luisa Mansego ${ }^{1-3}$, Fermín I. Milagro $^{1-3}$, \\ Amelia Marti ${ }^{1,3,4}$, J. Alfredo Martínez ${ }^{1-4}$ \\ ${ }^{1}$ Department of Nutrition, Food Science and Physiology, University of \\ Navarra, Spain; ${ }^{2}$ Centre for Nutrition Research, University of Navarra, \\ Spain; ${ }^{3}$ CIBER Fisiopatologia de la Obesidad y Nutricin (CIBERobn), \\ Instituto de Salud Carlos III, Spain; ${ }^{4}$ Navarra Institute for Health Research, \\ IdiSNA, Spain
}

Background: Epigenetics, including non-coding RNA, plays a role in the onset and development of obesity and related comorbidities. The aim of the present study was to evaluate the association between the DNA methylation levels of CpG sites located in miRNA coding sequences and childhood obesity.

Materials and Methods: Genomic DNA isolated from white blood cells of 24 children (12 obese; 12 non-obese) from the GENOI study was hybridized in a $450 \mathrm{~K}$ methylation microarray. Several CpGs whose DNA methylation levels were statistically different between obese and non-obese were further validated by Sequenom MassArray ${ }^{\circledR}$ EpiTyper ${ }^{\mathrm{TM}}$ in 95 children from the same study. Putative target genes for selected miRNAs were identified using miRBase.

Results: Microarray analysis identified 16 differentially methylated CpGs between both groups ( 6 hypermethylated and 10 hypomethylated). The network analysis performed with DIANA mirPath and IPA software featured 19 welldefined obesity-relevant biological pathways from KEGG database, including TGF-beta, neurotrophin, insulin, mitogen-activated protein kinases (MAPK) and Wnt signaling pathways. MassArray ${ }^{\circledR}$ EpiTyper $^{\mathrm{TM}}$ validation identified 3 regions located in or near miR-1203 (cg11210138), miR-412 (cg21230021) and miR-216A (cg24343835) coding regions differentially methylated between obese and non-obese children. The predictors of the model explained up to $44 \%$ of the variation of the BMI-SDS in the case of cg11210138 located in miR-1203 sequence, $41 \%$ in the case of $\operatorname{cg} 21230021$ (miR-412), and $40 \%$ in the case of cg24343835 (miR-216A).

Conclusions: The current investigation identified $3 \mathrm{CpG}$ sites located in coding regions of three miRNAs (miR-1203, miR-412 and miR-216A) that were differentially methylated between obese and non-obese children, suggesting a potential role of miRNA epigenetic regulation in childhood obesity. 


\section{P20}

\section{Influence of Oxygen Consumption on IL6 Promoter Methylation in PBMC from Obese Subjects with Sleep Apnea-Hypopnea Syndrome}

Amaya Lopez-Pascual 1,2, Pedro González-Muniesa ${ }^{1-4}$, Arrate Lasa ${ }^{3,5}$, Maria Puy Portillo ${ }^{3,5}$, Fernando Arós ${ }^{3,6}$, J. Alfredo Martínez $^{1-4}$

${ }^{1}$ Department of Nutrition, Food Science and Physiology, University of Navarra, Spain; ${ }^{2}$ Centre for Nutrition Research, University of Navarra, Spain; ${ }^{3}$ CIBER Fisiopatologia de la Obesidad y Nutricion (CIBERobn), Instituto de Salud Carlos III, Spain; ${ }^{4}$ Navarra Institute for Health Research, IdiSNA, Spain; ${ }^{5}$ Nutrition and Obesity Group/Department of Nutrition and Food Science, University of the Basque Country and Lucio Lascaray Research Center, Spain; ${ }^{6}$ Department of Cardiology, University Hospital of Alava, Spain

Background: DNA methylation is an epigenetic modification that effectively regulates gene expression, usually via gene silencing, which may contribute to the onset of many multifactorial diseases. The aim of the present study was to analyze the influence of basal oxygen consumption on gene promoter methylation and protein secretion of inflammatory markers in peripheral blood mononuclear cells from obese subjects with sleep apnea-hypopnea syndrome (SAHS), which due to their pathological conditions suffer different degrees of body hypoxia.

Methods: Blood samples were taken from 44 obese subjects with SAHS. Participants were categorized according to their basal low or high oxygen consumption ( $\leq 3.5$ and $3.5 \mathrm{ml} \cdot \mathrm{kg}-1 \cdot \mathrm{min}-1$, respectively). DNA methylation levels of the promoter region of four genes: LINE1, TNF, SERPINE1 and IL6, were evaluated using a methylation-sensitive high resolution melting approach after bisulfite modification. Serum proteins were measured by commercial EnzymeLinked Immunosorbent Assay kits.

Results: The methylation levels of the CpG Island found in the distal promoter sequence of human $I L 6$ were statistically reduced in low compared to those subjects on the high oxygen consumption group. Serum IL-6 was significantly higher in low oxygen consumption group. Finally, an age-related loss of DNA methylation of TNF promoter region was found.

Conclusions: These findings suggest a potential role of oxygen in the epigenetic regulation of inflammatory genes. The positive association between basal oxygen consumption and IL6 methylation could help clarifying the epigenetic mechanisms underlying mild chronic inflammatory diseases such as obesity and SAHS. Moreover, TNF methylation levels suggest a potential role of epigenetic modifications in the development of age-related inflammatory diseases. 


\section{P21}

\section{Maresin 1 Improves the Inflammatory State in White Adipose Tissue of $o b / o b$ Mice}

Leyre Martinez-Fernandez ${ }^{1,2}$, Pedro Gonzalez-Muniesa ${ }^{1-4}$, Laura M. Laiglesia ${ }^{1,2}$, J. Alfredo Martinez ${ }^{1-4}$, María J. Moreno-Aliaga ${ }^{1-4}$

${ }^{1}$ Department of Nutrition, Food Science and Physiology, University of Navarra, Pamplona, Spain; ${ }^{2}$ Centre for Nutrition Research, University of Navarra, Pamplona, Spain; ${ }^{3} \mathrm{CIBER}$ Fisiopatologia de la Obesidad y Nutricion (CIBERobn), Instituto de Salud Carlos III (ISCIII), Madrid, Spain; ${ }^{4}$ Navarra Institute for Health Research, (IdiSNA), Pamplona, Spain

Introduction: White adipose tissue (WAT) dysfunctional expansion in obesity leads to a low-grade chronic inflammation that ultimately contributes to the development of related disorders such as type 2 diabetes and cardiovascular diseases. This inflammatory state is associated with the rise in pro-inflammatory mediators and a reduction of anti-inflammatory adipocytokines released by WAT. N-3 polyunsaturated fatty acids display anti-inflammatory actions ascribed in part to the synthesis of pro-resolving lipid mediators derived from these fatty acids (i.e. resolvins, protectins and maresins). These lipid mediators are known to act as 'braking signals' of the unresolved inflammation and could provide a novel therapeutic strategy for treating obesity-induced diseases.

Objective: The aim of this study was to examine the effects of the docosahexaenoic acid (DHA)-derived lipid mediator maresin 1 (MaR1) on the expression of proinflammatory/anti-inflammatory adipocytokines and chemokines in the WAT of $o b / o b$ mice.

Methods: Male $o b / o b$ mice were treated (i.p) with saline or Maresin-1 (2 $\mu \mathrm{g} / \mathrm{kg}$ ) during 20 days. $M C P-1, I L-6, T N F-\alpha, I L-1 \beta, D P P-4$, Adiponectin and $I L-10$ mRNA expression was determined by RT-PCR.

Results: MaR1 administration decreased the mRNA expression levels of the chemoattractant protein $M c p-1$ and the pro-inflammatory cytokines $I l-1 \beta$ and Tnf- $\alpha$. On the contrary, gene expression levels of the anti-inflammatory cytokine Il-10 and Adiponectin mRNA were upregulated in MaR1-treated mice. Furthermore, the mRNA expression of the enzyme $D p p-4$, whose inhibitors exert glucose regulatory actions by prolonging the effects of incretins, was downregulated in $o b / o b$ mice treated with MaR1.

Conclusions: These data show that MaR1 is capable of modulating the expression of adipocytokines involved in the regulation of inflammation and insulin sensitivity, which could contribute to the insulin-sensitizing effects of this DHA-derived lipid mediator in obesity. 

Related to Lipid Profile and ox-LDL in Adolescents with Cardiovascular Risk Factors

Maria Horst, Carla Cristina Moraes, Cristiane Cominetti

Faculty of Nutrition, Federal University of Goias, Brazil

Objectives: To evaluate the additive effect of the polymorphisms rs18011282 (Pro12Ala) in PPARG; rs429358 + rs7412 (genotypes E2, E3 e E4) in APOE; rs1801133 and rs1801131 (C677T and A1298C, respectively) in MTHFR on serum lipid profile and oxidized LDL (ox-LDL) concentrations of adolescents with cardiovascular risk factors.

Methods: Cross-sectional study, with 115 Brazilian adolescents (aged 10-19). Questionnaires on socioeconomic data, lifestyle, medical history and food consumption were applied. Plasma ox-LDL concentration was determined through Elisa assay and serum lipid profile, by classic methodology. Genotyping was carried out by a fluorescence-based competitive allele-specific PCR. Multiple linear regression analysis and multivariate analysis of variance were performed to evaluate the additive effect of allelic variations on the evaluated biomarkers.

Results: Mean age (SD) was 13.9 (2.4) years; 58.3\% ( $n=67)$ were female; $69.6 \%(n=80)$ presented overweight and $53.9 \%(n=62)$ had dyslipidemia. The main results showed that Ala carriers ( $r$ 18011282) showed higher $(p=0.05)$ triacylglycerol and VLDL serum concentrations compared to non-carriers. For the ApoE gene polymorphisms, serum triacylglycerol and VLDL levels were marginally higher $(\mathrm{p}=0.1)$ in the E4-allele carriers compared to non-carriers. For MTHFR, the presence of $\mathrm{T}$ allele ( $\mathrm{rs} 1801133)$ was associated with higher $(\mathrm{p}=$ 0.05 ) plasma ox-LDL. However, the multiple linear regression analysis revealed no significant interaction $(p=0.05)$ between the four polymorphisms evaluated (additive effect) in the prediction of serum lipid profile and plasma ox-LDL.

Conclusions: The four polymorphisms were related to some cardiovascular risk biomarkers in adolescents when evaluated individually, however, when analyzed altogether they failed to predict alterations in lipid profile. 


\section{P23}

\section{The Small Food Additive Molecule Acetoin Triggers Biofilm} Formation by Prebiotic Bacteria

Noa Ben-Ishay ${ }^{1,2}$, Moshe Shemesh ${ }^{1}$

${ }^{1}$ Department of Food Quality and Safety, Institute for Postharvest Technology and Food Sciences, Agriculture Research Organization, The Volcani Center, Bet-Dagan, Israel; ${ }^{2}$ The Robert H. Smith Faculty of Agriculture, Food and Environment, The Institute of Biochemistry, Food Science and Nutrition, The Hebrew University of Jerusalem, Rehovot, Israel

Food products are often enriched by different food additives which may improve organoleptic and sensory characteristics of the products. Those additives can affect the physiology of many bacteria associated to human health, and affect development of multicellular community of bacterial cells known as a biofilm. Among those additives, there are important small molecules such as acetoin which can significantly improve the flavor of different food products. Acetoin (3-hydroxy-2-butanone) is a pale to yellowish liquid with a pleasant yogurt creamy odor and a fatty butter taste. It is a substance generally recognized as safe (GRAS) and mainly used in food industries to enhance the flavor of their products. This volatile compound widely exists in nature. Some microorganisms, higher plants, insects, and higher animals have the ability to synthesize acetoin using different enzymes and pathways under certain circumstances. Our preliminary results indicate that health promoting prebiotic bacteria such as Bacillus subtilis develop into complex bundle during growth in the presence of acetoin. $B$. subtilis cells express high levels of the extracellular matrix components, in response to acetoin, which are crucial for biofilm formation. We thus hypothesize that food additives such as acetoin trigger biofilm formation by health promoting bacterial species. It is believed that results of our study will open opportunities to important innovations in the food industry, such as development of novel nutritional supplements and prebiotic combination to promote biofilm formation by health promoting bacteria and to improve food quality. 


\title{
P24
}

Impact of Thermal Processing of Bovine Lactoferrin on Gut Microbiota

\author{
Alice M. Moscovici ${ }^{1}$, Peter Skuse ${ }^{2}$, Paul Cotter ${ }^{2}$, Uri Lesmes ${ }^{1}$ \\ ${ }^{1}$ Department of Biotechnology and Food Engineering, Technion - Israel \\ Institute of Technology, Israel; ${ }^{2}$ Food Biosciences Department, Teagasc \\ Food Research Centre, Ireland
}

Gut microbiota is increasingly recognized as a pivotal factor in shaping and maintaining human health and well-being. Concomitantly, there is a tremendous interest in food processing ramifications, and specifically thermal processing, on food digestibility and in turn on the composition and function of consumer endogenous microbiota. Studies have demonstrated that thermal processing, including Maillard reaction which may occur in protein-carbohydrate mixtures, alters proteolysis. However, gaps exist in understanding how processing can be harnessed to impact protein digestive fate, particularly the gut microbiome.

This work focused on prebiotic fructo-oligosaccharides (FOS) and bovine lactoferrin, a precursor for various bioactive peptides, e.g. antimicrobial, immune-modulating and bifidogenic. Lactoferrin was processed via controlled dry-heating $\left(60^{\circ} \mathrm{C}, 79 \% \mathrm{RH}\right)$ with and without FOS to isolate the Maillard reaction effect from the thermal one. Samples were predigested in an in vitro adult gastrointestinal model and dialyzed to partially simulate removal of low MW molecules. Obtained digesta was fed to an ex vivo batch colon model inoculated with adult human feces to study fermentability. Samples were collected at different time points and analyzed for microbial composition by 16S rRNA sequencing using Illumina Miseq. Lactoferrin digesta, i.e. native, heated or conjugated with FOS (MLf) led to alterations in gut microbiota, but produced a generally similar bacterial profile. This included enrichment in Enterobacteriaceae and a notable decrease in Bacteroides compared to FOS. In addition, an increase in Acidaminococcus was detected at fermentation end point which was not in the FOS control. Moreover, Bifidobacteriaceae/Bifidobacterium relative abundance was lower in the MLf vessel compared to FOS.

Overall, this study shows controlled processing of lactoferrin may have some effect on gut microbiota, and offers a holistic approach to rational development of products designed to benefit consumers. 


\title{
P25 \\ Influence of Gestational Diabetes on Metabolite Concentrations in Human Colostrum
}

\author{
Ilana Chertok ${ }^{5}$, Nissim Silanikove ${ }^{2}$, Smadar Eventov-Friedman ${ }^{3}$, \\ Zelalem Haile ${ }^{4}$, Nurit Argov-Argaman ${ }^{1}$
}

${ }^{1}$ Robert H. Smith Faculty of Agriculture, Food and Environment, Hebrew University, Israel; ${ }^{2}$ Institute of Animal Science, Hebrew University, Israel; ${ }^{3}$ Department of Neonatology, Hebrew University, Israel; ${ }^{4}$ Department of Social Medicine, Ohio University, USA; ${ }^{5}$ College of Health and Human Services, University of North Carolina at Charlotte, USA

Background: Breastfeeding is associated with protection against the development of type 2 diabetes in women with a history of gestational diabetes mellitus (GDM), in a dose dependent manner. Thus, longer duration and higher intensity of breastfeeding is recommended for women with GDM although women with GDM often have lower rates of breastfeeding and face more challenges with early breastfeeding than women without diabetes. There is a paucity of information regarding the effect of GDM on the metabolite composition of colostrum. The metabolite concentrations of lactose and glucose indicate the transition to increased milk production known as lactogenesis II.

Objectives: A primary objective of the study was to examine and compare metabolite levels of glucose and lactose in colostrum of women with and without GDM.

Method: A prospective case-control study of postpartum women, 32 with GDM and 35 without GDM, was conducted to examine differences in colostral metabolite levels using enzymatic methods.

Results: Composition analysis revealed significantly lower concentrations of lactose at 72 hours postpartum in colostral samples of women with GDM compared to women with diabetes (5.8 versus $6.3, \mathrm{p}=0.043$ ). Likewise, glucose levels were significantly lower in the colostral samples of women with GDM (142.4 versus 170.8, $\mathrm{p}=0.027$ ).

Discussion: Significantly lower levels of colostral lactose and glucose among women with GDM compared to those without GDM suggests a delay in lactogenesis II among women with GDM which may suggest a biological mechanism associated with lower breastfeeding rates among women with GDM. Early and frequent breastfeeding and pumping among women with GDM can facilitate the transition to lactogenesis II and promote breastfeeding among this at-risk population.

Conclusion: Differences in colostral concentrations of lactose and glucose between women with and without GDM suggests that women with GDM experience delayed lactogenesis II which may negatively impact breastfeeding outcomes in women with GDM. 


\title{
P26
}

\section{Expression of miRNAs Related to Obesity and Diabetes in Human Milk}

\author{
Yaffa Elbaum Shiff 1 , Regina Golan-Gerstl', Shimon Reif ${ }^{2}$
}

${ }^{1}$ The School of Nutritional Sciences, The Hebrew University, Israel;

${ }^{2}$ Department of Pediatrics, Hadassah Ein-Kerem Medical Center, Jerusalem, Israel

Introduction: Several different epidemiological studies have demonstrated that breastfeeding can reduce the rate of obesity and diabetes. We, as well as others, have demonstrated that human milk contains exosomes, which have the ability to transfer miRNA intracellular by fusion. Moreover, we found that the fat layer in milk is rich in miRNA.

We hypotheses that human milk contains beneficial miRNAs that can be involved in the preventive effect of milk in childhood diseases such as obesity and diabetes.

Methods: Milk miRNAs were isolated from the exosomes in the skim milk and from the fat globules in the lipid layer of human milk. Next sequencing generation (NGS) and Real Time PCR were performed to study the profile expression of miRNAs in milk.

Results: We have partially determinate the profile expression of miRNA in milk of 15 mothers from term and preterm babies at different periods of lactation. We have identified several known important miRNA in the skim and fat fraction of human milk at different time of lactation. We demonstrated that miRNAs related to pancreatic genesis and insulin resistance development, such as mir-375 and mir-320 have different levels of expression at different periods of lactation, as well between different mothers.

Conclusion: We found expression of beneficial miRNAs in milk that can regulate target genes with potential molecular and physiological functions in development of obesity and diabetes. Those miRNA may account for the observed protective effect of human milk against obesity and diabetes. Our results have experimental basis in attempt to add beneficial miRNAs carried by exosomes and fat globules from milk to infant formula. 


\section{P27}

The Study of the Association of Genetic Polymorphisms with Obesity in Children of Preschool and School Age:

\section{A Pilot Study}

\section{Nataliya Shilina, Elena Sorokina, Adelya Safronova}

Igor Kon Laboratory of Age-Related Nutritiology, Institute of Nutrition, Russia

Introduction: Childhood obesity is increasing rapidly worldwide. The variation in the fat mass and obesity - associated (FTO) gene and the $\beta 3$ adrenoreceptor (ADRB3) gene has provided the robust associations with common obesity. However the association of FTO single nucleotide polymorphism (SNP) rs9939609 with BMI first becomes evident at early adolescence. Our aim was to study the age peculiarities of association of FTO SNP rs9939609 and ADRB3 SNP rs4994 with obesity in Russian children of Moscow region.

Methods: We studied 191 children, 3 to 10 years of age, who underwent genotyping for FTO rs 9939609 and ADRB3 rs4994 SNPs and were measured for height and weight.

Results: In all children observed the frequency of allele T of FTO rs9939609 SNP was $61.7 \%$, allele A - 38.3\%. The frequency of obesity risk allele (A) in overweight preschool age children (3-6 years of age) was 7.1\% higher (OR-1.43; Cl [0.47-4.39], $\mathrm{p}=0.53)$, and in overweight school age children (7-10 years of age) was $16 \%$ higher (OR-1.94; $\mathrm{Cl}[0.79-4.76], \mathrm{p}=0.14$ ) than that of normal weight children (NWC) but not significantly (NS) in both cases. The frequency of Trp64 allele of $A D R B 3$ SNP rs4994 was $91.6 \%$, Arg64-8.4\%. The frequency of obesity risk allele $(64 \mathrm{Arg})$ in overweight children was $2.9 \%$ higher than that of NWC but NS (OR-1.37; Cl [0.49-3.86], p = 0.54). The frequency of 64Arg allele in overweight preschool age children did not differ from that of NWC (OR-1.03; Cl [0.16-6.63], $\mathrm{p}=0.98)$. In overweight school age children the frequency of 64Arg was $4.2 \%$ higher than that of NWC (OR-1.52; Cl [0.38- 6.03], $\mathrm{p}=0.55)$.

Conclusion: The association of alleles (A) of FTO rs9939609 SNP and (64Arg) of $A D R B 3$ rs4994 SNPs with obesity risk increased with increasing age of children but still was not significant at age 10 . 
Microbiota Composition of Ugandan Children Following Vitamin A Supplementation

\author{
Elina Manusevich Wiseman ${ }^{1}$, Omry Koren ${ }^{2}$, Ram Reifen ${ }^{1}$ \\ ${ }^{1}$ Food and Environment, The Robert H. Smith Faculty of Agriculture, \\ The Hebrew University of Jerusalem, Israel; ${ }^{2}$ Microbiology, The Faculty \\ of Medicine, Bar-Ilan University (Safed campus), Israel
}

Introduction: Vitamin A deficiency (VAD) affects about 250 million preschool children, mainly in developing countries, where it increases morbidity and death from severe infections. Vitamin A (VA) is considered a comprehensive immunomodulator. In first-line defence, VAD impacts the integrity of gut mucosal barrier and the secretion of sIgA, whereas in the adaptive response, VAD adversely affects the generation of T cells, especially Th17, iTreg and FoxP3+Treg. Microbiota is another entity associated with the immune system. Exposure to variety of appropriate bacteria in infancy is crucial for immunological development. The influence of the microbiota on both innate and adaptive phases overlaps some of VA effects. We examined the interaction between VA status and the microbiota. Up to now only a few animal model studies have been done and report a change in bacterial quantity and composition. We hypothesized that VA, not only act on immune cells directly, but also, influence the microbiota, which executes its effect on the immune system.

Objective: To investigate microbiota characteristics before and after vitamin A supplementation (VAS) and explore the association between VA status and the microbiota, on the basis of mutual immune system outcomes.

Methods: A total of 100 Ugandan children were recruited at 6 months of age. Stool samples were collected at 4 time points: baseline - before routine VAS, a day, a week and a month after VAS. Stool samples of 67 children who have completed a full time-series collection were examined using 16S rRNA gene sequencing, followed by data analysis using QIIME. At baseline, health and nutritional data, anthropometry and blood sample were collected. The latter was also collected a month after VAS and analysed for VA levels using HPLC.

Results and Conclusions: Quantitative and qualitative analyses revealed: microbiota diversity and structure following VAS and additionally VA status. 


\section{P29}

Transcriptome-Based Identification of New Anti-Inflammatory Properties of the Olive Oil Hydroxytyrosol in Vascular Endothelial Cell Under Basal and Proinflammatory Conditions

Maria Annunziata Carluccio ${ }^{1}$, Marika Massaro ${ }^{1}$, Egeria Scoditti ${ }^{1}$, Nadia Calabriso ${ }^{1}$, Mariangela Pellegrino ${ }^{3}$, Rosanna Martinelli, Valentina Gatta ${ }^{4}$, Raffaele De Caterina ${ }^{4}$

${ }^{1}$ Institute of Clinical Physiology, CNR, Italy; ${ }^{2}$ Department of Biochemistry and Medical Biotechnologies, University of Salerno, Italy; ${ }^{3}$ Department of Biological and Environmental Science and Technology, University of Salento, Italy; ${ }^{4}$ Department of Neuroscience and Imaging, G. d' Annunzio University, Italy

Introduction: The extravirgin olive oil polyphenol hydroxytyrosol (HT) is regarded as responsible for the beneficial effects associated with olive oil consumption. However, the underlying molecular basis remains incompletely defined. Using a DNA microarray technology we investigated the early gene expression profile of human vascular endothelium conditioned by HT under basal and pro-inflammatory conditions.

Methods: Human umbilical vein endothelial cells (HUVECs) were treated

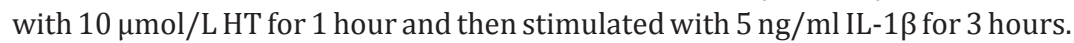
Total RNA was extracted and gene expression profile performed with an Agilent Microarray covering 41000 genes and transcripts. Raw data were processed with the GeneSpring ${ }^{\circledR} 10$ software and differentially expressed RNA identified using Benjamini and Hochberg False Discovery Rate with a P-value 0.05. Functional and network analyses were identified by the Ingenuity Pathways Analysis.

Results: Fixing a significance threshold at 1.5 fold of change (FC), HT, per se, changed the expression of 707 genes, down-regulating 269 and up-regulating 438. IL-1 stimulation changed the expression of 2389 genes, down-regulating 1120 and up-regulating 1269. Treatment with HT before IL-1 stimulation significantly affected the expression of 599 IL-1-deregulated genes. The application of the Ingenuity pathway analysis software allowed us to pinpoint immunological-, inflammatory- and metabolic-related pathways as the most affected. Under basal conditions HT down-regulated the expression of several chemokines and related receptor including those for the chemokine (C-C motif) ligand 2 and 20 and tumor necrosis factor receptor associated factor (TRAF)-1. Under pro-inflammatory stimulation, HT pre-treatment again counter-regulated gene expressions involved in inflammatory and proliferative pathways.

Conclusions: Endothelial exposure to HT affects endothelial gene expression under both basal and pro-inflammatory settings. Most of HT-regulated genes were connected to angiogenesis, inflammation and atherogenesis. The unbiased identification of novel genes regulated by HT treatment improves our understanding of mechanisms by which olive oil may prevent or attenuate human inflammatory diseases. 


\title{
P30
}

Anti-Inflammatory Nutrigenomic Effects of Hydroxytyrosol in Human Adipocytes - Protective Mechanisms of Mediterranean Diets in Obesity-Related Inflammation

\author{
Egeria Scoditti ${ }^{1}$, Mariangela Pellegrino ${ }^{1,2}$, Marika Massaro ${ }^{1}$, \\ Maria Annunziata Carluccio ${ }^{1}$, Nadia Calabriso ${ }^{1}$, Carlo Storelli ${ }^{2}$, \\ Raffaele De Caterina ${ }^{3}$ \\ ${ }^{1}$ Institute of Clinical Physiology, CNR, Italy; ${ }^{2}$ Department of Biological \\ and Environmental Science and Technology (Disteba), University of \\ Salento, Italy; ${ }^{3}$ Department of Neuroscience and Imaging, G. d'Annunzio \\ University, Italy
}

Introduction: Obesity is associated with increased risk of cardio-metabolic disorders. Excess adiposity results in oxidative stress and dysregulation of adipokine production, and is accompanied by macrophage infiltration in the adipose tissue, contributing to local and systemic inflammation. We investigated the effects of the Mediterranean diet olive oil polyphenolic antioxidant hydroxytyrosol (HT) on the expression of adipokines regulated by tumor necrosis factor (TNF)-alpha in human Simpson-Golabi-Behmel Syndrome (SGBS) adipocytes, exploring underlying mechanisms.

Methods: SGBS adipocytes were treated with 1-10 $\mu \mathrm{mol} / \mathrm{L}$ HT for $1 \mathrm{~h}$ before $10 \mathrm{ng} / \mathrm{ml}$ TNF-alpha for $24 \mathrm{~h}$. Adipokines mRNA expression and levels in the culture medium were measured by real time PCR and EIA, respectively. Effects on the activation of the transcription factor NF- $\kappa$ B p65 subunit were determined by ELISA and Western blotting. The production of reactive oxygen species (ROS) was detected by the fluorophore dichlorofluorescein diacetate.

Results: Under pro-inflammatory conditions induced by TNF-alpha, adipocytes increased expression and medium levels of monocyte chemoattractant protein (MCP)-1, chemokine (C-X-C Motif) Ligand 10, interleukin (IL)-1 $\beta$, IL-6, vascular endothelial growth factor (VEGF), plasminogen activator inhibitor (PAI)-1, cyclooxygenase (COX)-2, macrophage colony-stimulating factor (M-CSF), metalloproteinase (MMP)-2, superoxide dismutase (SOD) and glutathione peroxidase (GPX), and the surface expression of intercellular adhesion molecule (ICAM)-1, and reduced the release of adiponectin (APN) and the expression of endothelial nitric oxide synthase (eNOS). Pretreatment with HT significantly inhibited TNF- $\alpha$-induced expression and/or release of pro-inflammatory cytokines, and reverted TNFalpha-mediated inhibition of APN and eNOS. HT also prevented the TNF-alpha-induced binding activity and nuclear translocation of $\mathrm{NF}-\kappa \mathrm{B}$ and ROS production.

Conclusions: Our data suggest that HT, at concentrations achievable through Mediterranean diets, can modulate the expression and secretion of adipokines in adipocytes through a mechanism involving reduction in oxidative stress and NF- $\kappa$ B inhibition. By such mechanisms, HT may therefore blunt macrophage recruitment and improve adipose tissue inflammation, possibly preventing the activation of pathways involved in obesity-related diseases. 


\section{P31}

\section{Phosphodiesterase5A Up-Regulation in Vascular} Endothelium Under Pro-Inflammatory Conditions: A Newly Disclosed Anti-Inflammatory Activity for the w-3 Polyunsaturated Fatty Acid Docosahexaenoic Acid

Marika Massaro ${ }^{1}$, Egeria Scoditti ${ }^{1}$, Mariangela Pellegrino ${ }^{2}$, Nadia Calabriso ${ }^{1}$, Maria Annunziata Carluccio ${ }^{1}$, Carlo Storelli², Raffaele De Caterina ${ }^{3}$

${ }^{1}$ Institute of Clinical Physiology, CNR, Italy; ${ }^{2}$ Department of Biological and Environmental Science and Technology, University of Salento, Italy; ${ }^{3}$ Department of Neuroscience and Imaging, G. d'Annunzio University, Italy

Introduction: Phosphodiesterase (PD)5A (PDE5A) catalyzes the hydrolysis of cGMP into GMP, thus curtailing NO signaling and favoring inflammation. The $\omega$-3 fatty acids docosahexaenoate (DHA) and eicosapentaenoate (EPA) are considered health-promoting nutrients. However, molecular mechanisms underlying their final effects remain incompletely understood. We therefore investigated whether inflammatory stimuli known to be involved in inflammatory endothelial dysfunction and angiogenesis affect endothelial PDE5A expression, and whether cell exposure to DHA alters such expression.

Methods: Human umbilical vein endothelial cells (HUVECs) were treated with increasing concentrations of the inflammatory and pro-angiogenic stimuli interleukin (IL)-1b, tumor necrosis factor (TNF) $\alpha$, IL-6, and vascular endothelial growth factor (VEGF) for 0-24 hours. After this time, PDE5A protein and mRNA expression were assessed by Western and qPCR, while the activation of Nuclear factor (NF)- $\kappa \mathrm{B}$ and Activator Protein (AP)-1, in terms of RelA-, c-Fos-, pospho-cJun-, Fos-B-, and Fra-1-DNA binding, were assessed by trans-activation assays. To evaluate DHA effect on PDE5A expression, HUVECs were treated with 0-50 $\mu \mathrm{mol} / \mathrm{L}$ DHA for 48 hours before stimulation.

Results: PDE5A protein and mRNA expression increased significantly after stimulation with $10 \mathrm{ng} / \mathrm{ml} \mathrm{IL-1b}$ and TNF $\alpha$ (P = 0.01 vs. control). DHA treatment of HUVECs for 48 hours before cytokine stimulation reduced PDE5A induction at the protein and mRNA levels ( $\mathrm{P}=0.05 \mathrm{vs}$. IL- $1 \mathrm{~b}$ and TNF $\alpha$ ) and, correspondently, DNA binding of RelA, cFos, pospho-c-Jun and Fra-1 induced by IL-1b (for all, P = 0.05 vs. IL-1b).

Conclusions: Pro-inflammatory, but not pro-angiogenic stimuli, induce PDE5A expression in the endothelium, with the involvement of inflammatory signaling. DHA reduces PDE5A induction interfering with both NF- $\kappa$ B and AP-1 activation. Since PDE5A inhibitors are now approved for use in erectile dysfunction and pulmonary hypertension and have a potential in treating other disease states featuring endothelial dysfunction, the reduction by DHA of inflammation-mediated expression of PDE5A may favorably impact the NO/sGC/cGMP axis and reproduce the therapeutic potential of PDE5 inhibitors. 


\section{P32}

\section{Vitamin A and Epigenetics}

Alona Metz ${ }^{1}$, Regina Golan-Gerstl ${ }^{2}$, Yaffa Elbaum Shiff ${ }^{3}$, Ram Reifen ${ }^{1}$

${ }^{1}$ Food and Environment, The Robert H. Smith Faculty of Agriculture, The Hebrew University of Jerusalem, Israel; ${ }^{2}$ Department of Pediatrics, Hadassah Medical Center, Jerusalem, Israel; ${ }^{3}$ The School of Nutritional Sciences, The Hebrew University, Israel

Introduction: Epigenetics is defined as heritable changes in gene expression that are not attributable to alterations in the DNA sequence. RNA interference through miRNA is one mechanism of epigenetics, which inhibits translation of mRNA into protein. Epigenetics is affected by environmental factors, including diet and nutrition that can influence physiologic and pathologic processes, these terms as nutritional epigenetics.

Vitamin A (VA) is an example for a nutrient that can lead to nutritional epigenetic changes. VA is essential for maintaining proper embryonic development and growth, intact immune system activity, tissue differentiation, gut integrity and the visual cycle.

It regulates gene transcription through interaction to its nuclear receptor. The action mechanism of VA is highly analogous to that of steroid hormones which usually act by gene activation/deactivation. Little is known regarding the impact of VA on miRNA levels. The aim of the study is to explore this impact in VA sufficient (VAS) and deficient (VAD) breastfeeding mothers in Uganda.

Methods: 100 lactating mothers were recruited for the study. 88 breast milk samples were collected and examined by HPLC for VA levels. The samples from VAS and VAD mothers were further evaluated for VA-related miRNAs levels by qPCR.

Results and Conclusions: The results of this study may indicate on VA as an epigenetic modulator in critical developmental time points. Importantly, specific epigenetic patterns could serve as potential markers for VA status. 

Growth and Survival of Probiotic Health Promoting Bacteria $\underline{\text { Sagit Yahav }}^{1,2}$, Moshe Shemesh ${ }^{1}$

${ }^{1}$ Department of Food Quality and Safety, Institute for Postharvest Technology and Food Sciences, Agriculture Research Organization, The Volcani Center, Israel; ${ }^{2}$ The Institute of Biochemistry, Food Science and Nutrition, The Hebrew University of Jerusalem, Israel

Introduction: Probiotics are live microbial feed supplements which beneficially affect the host by improving its intestinal microbial balance. Due to their perceived health benefits, probiotics bacteria are increasingly incorporated into a variety of food and drink products. To assure the beneficial effects in the body, these organisms must survive during food processing, storage and the passage through the upper gastrointestinal tract (GIT) and arrive alive to its site of action. However, studies have shown low survival of probiotic bacteria in the final food product and a considerable loss in viability when they encounter the very acidic conditions of the stomach and high bile concentration in the small intestine. Bacillus subtilis, an endospore-forming bacterium, is not normally found in the GIT but have also been shown to be effective in keeping a favorable balance of microflora in the GIT. B. subtilis spores are resistant to extreme environmental condition, therefore many live spores administered orally may reach the intestine and induce beneficial effects. In addition, B. subtilis produces extracellular matrix that protects it from stressful environmental conditions.

Results and Conclusions: Our preliminary results indicate that probiotic bacteria are able to grow in the presence of $B$. subtilis under conditions that increase the production of the extracellular matrix. We thus hypothesize that extracellular matrix produced by $B$. subtilis may protect other probiotic bacteria. Hence, it can be used as a potential vehicle for the delivery of viable probiotic cells. It is believed that results of our study will provide a novel technique in using a natural system for maintaining and delivery of probiotics to human. 


\section{P34}

Isoflavone Metabolizing Phenotypes Dictated by Diet in Men with Asymptomatic Prostate Cancer

Jennifer Ahn-Jarvis ${ }^{1}$, Elizabeth Granger ${ }^{2,4}$, Kenneth Riedl 1,4, $^{3,4}$ Steven Clinton ${ }^{2,4}$, Steven Schwartz ${ }^{3,4}$, Yael Vodovotz ${ }^{3,4}$

${ }^{1}$ College of Public Health, The Ohio State University, USA; ${ }^{2}$ College of Medicine, Department of Internal Medicine, Division of Medical Oncology, The Ohio State University Wexner Medical Center, USA; ${ }^{3}$ Department of Food Science and Technology, The Ohio State University, USA; ${ }^{4}$ Comprehensive Cancer Center, The Ohio State University, USA

Previous studies demonstrate mixed effects on health outcomes with soy interventions. Isoflavone metabolites of daidzein and genistein were quantified in urine during a 20 week intervention in 32 men with asymptomatic prostate cancer and isoflavonoid metabolite profiles clustered into four phenotypes. Cluster 1 was characterized as high in genistein and daidizein; cluster 2 was high in O-desmethylangolensin (ODMA), cluster 3 having both equol and ODMA; and cluster 4 enriched in equol. Given that isoflavone metabolizing phenotypes have been associated with obesity and conversely with health promotion, we hypothesize that differences in nutrient profiles from diet dictate differences in isoflavone metabolite profiles which impact health outcomes among individuals. The primary objective was to examine the role of diet on the phenotypic expression of isoflavone metabolites. During this study, men consumed two slices/day (85 g/ slice) of soy bread containing $\sim 60 \mathrm{mg}$ of isoflavones. A Food Frequency Questionnaire and 3-day diet records were used to assess diet. We also measured biomarkers of inflammation (IL-6) and obesity (leptin). No significant differences were observed in total energy or macronutrients consumed yet significant differences ( $\mathrm{p} \leq 0.05$ ) were observed in consumption of dietary B vitamins and minerals between cluster 1 and cluster 3. Similarly, food frequency questionnaires revealed calcium from dairy sources, alpha-carotene, glucose, and fructose intake were significantly different between these two clusters. Additionally, significant differences in dairy food consumption were observed among equol producing phenotypes (cluster 3 and 4) compared to those who did not produce equol (cluster 1 and 2). Moreover, significant reduction in IL-6 was observed in cluster $1(37 \%)$ and 3 (59\%) and significant increase in leptin was observed in cluster 2 (17\%). These differences suggest that diet has an important role in mediating the phenotypic expression of isoflavone microbial metabolites and possibly has downstream effects on biomarkers of inflammation and obesity. 
A Cereal Contaminant Affects Genome-Wide Expression in Kidney of Weanling Piglets

Daniela Marin ${ }^{1}$, Cornelia Braicu², Mihai Alexandru Gras', Gina Cecilia Pistol', Roxana Cojocneanu-Petric ${ }^{2}$, loana Berindan Neagoe ${ }^{2}$, Ionelia Taranu ${ }^{1}$

${ }^{1}$ Laboratory of Animal Biology, National Institute for Research and Development for Biology and Animal Nutrition, 077015, Balotesti, Ilfov, Romania; ${ }^{2}$ Research Center for Functional Genomics, Biomedicine and Translational Medicine, 'luliu Hatieganu' University of Medicine and Pharmacy, 400565 Cluj-Napoca., Romania

Ochratoxin A is a toxic fungal metabolite produced by Aspergillus or Penicillum fungi growing on a wide range of raw food commodities. The aim of the present paper was to investigate the nefrotoxic effect of OTA as the guidance value recommended by EU (50 ppb) in the kidney of weanling piglets. The wide genome expression was analysed using a DNA microarray. The microarray analysis showed that OTA contaminated diet induced significant changes on global transcriptome in kidney of intoxicated pigs. The microarray data identified 796 genes significantly different $(\mathrm{P}=0.005)$ expressed in kidney of pigs fed OTA contaminated diet compared to control; of these $38.94 \%$ (310 genes) were down-regulated and $61.06 \%$ (486 genes) up-regulated. Gene Ontology analysis has shown the alteration of the expression of genes involved in nephrotoxicity (eg: in renal necrosis/cell death, kidney failure, renal proliferation, renal dysplasia and hypoplasia) but also in general toxicity (eg. fatty acid metabolism, PXR/RXRactivation, cytochrome 450 panel, hypoxia-inducible factor signalling).

As shown by the microarray results, the dose of $50 \mathrm{ppb}$ of OTA can interfere with: i) canonical pathways (CD28 Signalling in T Helper Cells, Role of NFAT in Regulation of the Immune Response, Relaxin Signalling, IL-1 Signalling) ii) molecular and cellular function (cellular movement cellular function and maintenance, cellular growth and proliferation cellular assembly and organization, cell death and survival).

In summary, these results suggest that OTA as the guidance value recommended by EU ( $50 \mathrm{ppb}$ ) can alter the wide genome expression of weanling piglets in kidney. 


\section{P36}

Effect of Microbial and Myctoxin Co-Contamination on Wide Genome Profile of Porcine Epithelial Intestinal cells

Ionelia Taranu ${ }^{1}$, Cornelia Braicu², Gina Cecilia Pistol' ${ }^{1}$, Monica Motiu1, Daniela Eliza Marin ${ }^{1}$

${ }^{1}$ Animal Biology, National Institute for Research and Development for Biology and Animal Nutrition, Balotesti, Ilfov, Romania; ${ }^{2}$ Research Center for Functional Genomics, Biomedicine and Translational Medicine, 'Iuliu Hatieganu' University of Medicine and Pharmacy,

Cluj-Napoca, Romania

Environmental contaminants like heavy metals, perfluorated chemicals, aromatic hydrocarbons, mycotoxins, various microorganisms and many others counting among the xenobiotics that can contaminate the feed and food chain. Studies investigating the combine effect of two contaminants (e.g. microbial pathogen and mycotoxins) is little investigated in pig. Therefore the aim of the current study was to evaluate the effect of the in vitro exposure of porcine epithelial intestinal cells (IPEC-1) to a microbial and mycotoxin co-contamination in order to better understand the deeper changes in wide genome profile. Two contaminants were selected for this study which has both a high impact on pig, Escherichia coli (E. coli), one of the most frequent microorganism responsible for post weaning diarrhoea, and zearalenone (ZEA), mycotoxin which frequently contaminate animal feed and to which the pig reacts most sensitively among farm animal species. IPEC-1 cells were untreated (Control) or treated with E. coli $(7 \times 106 \mathrm{CFU} / \mathrm{ml})$, and ZEA $(25 \mu \mathrm{M})$, either alone or simultaneously for $1 \mathrm{~h}$. The expression profile of responsive genes was subjected to cluster and statistical microarray analyses by using Gene Spring GX v.11.5. The microarray analysis showed that gene up-regulation was the predominant effect after $1 \mathrm{~h}$ of contaminants treatment, individual or in combination ( $87 \%$ up-regulated genes). Among the functional groups of examined genes those related to signalling pathways were the most affected by ZEA and E. coli either alone or in combination. The up-regulation of transcriptional and growth factors, proliferation, cytokines, inflammatory response genes was observed. It was also noted that additive effects were not always registered. However, in the case of proliferation gene cluster, 20 genes were significantly down-regulated by E. coli treatment, 0 genes by ZEA, while 23 genes were downregulated by the combination E. coli and ZEA. These data provide a better understanding of the effects of microbial and mycotoxin co-contamination. 
Physical Activity Attenuates the Effect of the FTO rs9939609 Polymorphism on Obesity and Metabolic Syndrome in Lithuanian Adult Population

\author{
Alina Smalinskiene ${ }^{1}$, Janina Petkeviciene ${ }^{2}$, Jurate Klumbiene ${ }^{2}$, \\ Vaiva Lesauskaite ${ }^{1}$ \\ ${ }^{1}$ Institute of Cardiology, Medical Academy, Lithuanian University of \\ Health Sciences, Lithuania; ${ }^{2}$ Faculty of Public Health, Medical Academy, \\ Faculty of Public Health, Lithuanian University of Health Sciences, \\ Lithuania
}

Background: This study aimed to examine the associations between the fat mass and obesity associated (FTO) gene rs9939609 variant with obesity and and dietary intake or metabolic syndrome and interactions between FTO alleles and physical activity in Lithuanian adult population.

Methods: A cross-sectional health survey was carried out in randomly selected municipalities of Lithuania. The random sample was obtained from the lists of 25-64 year-old inhabitants registered at primary health care centres. The data from 1020 individuals (420 men and 600 women) were analysed. The single-nucleotide polymorphism, rs9939609, in FTO gene was assessed using a real-time polymerase chain reaction. 24-hour recall was used for evaluation of dietary habits. Information on physical activity was gathered by a standard questionnaire. Physical activity at work, travelling to and from work and at leisure time was evaluated.

Results: The carriers of the AA genotype had the highest mean values of body mass index (BMI) and waist circumference (WC). They had 1.72 time higher odds of obesity ( $\mathrm{P}=0.009)$ and 1.67 time higher odds of increased WC $(\mathrm{P}=0.013)$ than those with the TT genotype. Carriers of the T allele had lower prevalence of metabolic syndrome compared to carriers of the AA genotype (33.8\% 45 and $42.5 \%$ respectively; $\mathrm{P}=0.018$ ). No interaction between the rs 9939609 variant and 46 energy or dietary intakes on weight status was found. Significant effect of the interactions 'genotype $\mathrm{x}$ age' and 'genotype $\mathrm{x}$ physical activity' on BMI was demonstrated. The FTO rs9939609 polymorphism was associated with anthropometric parameters and metabolic syndrome in the younger age group (25-44 years) and in individuals having low level of physical activity.

Conclusions: The FTO rs9939609 variant was associated with weight status and metabolic syndrome in Lithuanian adult population. Age and physical activity modulated the effect of the FTO polymorphism. 


\title{
P38
}

\section{Antiinflammatory and Antihyperglycemic Effects of Quercetin and Quercetin-3-O-Glucoside in Cellular Models}

\author{
Ana Laura de la Garza ${ }^{1}$, Denys Torres-Villarreal', Fermín I. Milagro², \\ Rocío Ortíz-López ${ }^{3}$ \\ ${ }^{1}$ Faculty of Public Health and Nutrition and Centre for Research and \\ Development in the Health Sciences, Autonomous University of Nuevo \\ Leon, Mexico, Spain; ${ }^{2}$ Department of Nutrition, Food Science and \\ Physiology, Centre for Nutrition Research, University of Navarra, Spain; \\ ${ }^{3}$ Faculty of Medicine and Centre for Research and Development in the \\ Health Sciences, Autonomous University of Nuevo Leon, Mexico
}

Background: The association between the intake of flavonoid-containing foods and risk of several chronic diseases is being investigated due to the biological properties of flavonoids. Quercetin is the most abundant flavonol in nature (fruits, vegetables, leaves and grains) and also the most consumed flavonolThis study aims to investigate the effects of quercetin and quercetin-3-0-glucoside on inflammation and glucose uptake in 3T3-L1 adipocytes and CaCo2 cells.

Methods: The antiinflammatory effects of quercetin were evaluated in 3T3-L1 cells. 3T3-L1 preadipocytes were treated with quercetin (30 to $60 \mu \mathrm{M}$ ). CaCo2 cells were used to examine the effect of quercetin-3-0-glucoside (30 to 60 $\mu \mathrm{M})$ in the expression of genes involved in glucose uptake. mRNA expression of genes related to inflammation and glucose transporters was determined by RT-PCR.

Results: Treatment with quercetin $(60 \mu \mathrm{M})$ had significant effect on the mRNA expression of TNF $\alpha$ compared to the control cells. Likewise, quercetin decreased mRNA expression of MCP-1 in 3T3-L1 adipocytes in comparison with the control cells. On the other hand, in the presence of Na+, both SGLT1- and GLUT2-mediated glucose uptakes were downregulated in CaCo2 cells incubated with quercetin-3-0-glucoside.

Conclusions: Treatment of 3T3-L1 adipocytes with quercetin decreased the expression of genes involved in inflammation. Likewise, quercetin-3-0-glucoside downregulated the expression of genes related to inflammation and glucose uptake in $\mathrm{CaCo} 2$ cells. 


\section{P39}

\section{Fructose versus Sucrose Associated with Elevated Plasma Triglyceride and Glucose Levels: Preliminary Look to Developmental Programming}

Yagmur Kaya Betul Kisioglu, Reyhan Nergiz-Unal

Department of Nutrition and Dietetics, Faculty of Health Sciences, Hacettepe University, Turkey

Objectives: The aim of this study was to investigate the effects of maternal high fructose corn syrup versus sucrose intake on plasma glucose and triglyceride levels of the dams and pups.

Method: This study was carried out on Sprague Dawley strain female rats $(\mathrm{n}=14)$. After two weeks wash-out period the rats were randomly divided into two groups. Water including high fructose corn syrup (HFCS) or sucrose $0.2 \mathrm{~g} /$ $\mathrm{ml}(20 \% \mathrm{w} / \mathrm{v}))$ was administered for 12 weeks. After mating, the dietary manipulation continued during pregnancy and lactation periods. At the end of lactation period, blood was isolated from the rats under anesthesia and animals were immediately euthanized. The plasma glucose and triglyceride levels were analyzed by colorimetric method with a microplate reader.

Results: It was determined that mean plasma glucose level of dams in HFCS group was $316.6 \pm 99.44 \mathrm{mg} / \mathrm{dl}$ while in dams of the sucrose group was $105.7 \pm$ $18.39 \mathrm{mg} / \mathrm{dl}(\mathrm{P}=0.05)$. The mean plasma glucose level of pups in the HFCS group was $279.4 \pm 62.58 \mathrm{mg} / \mathrm{dl}$ while in pups of the sucrose group was $119.1 \pm 14.21$ $\mathrm{mg} / \mathrm{dl}(\mathrm{P}=0.05)$. It was also found that mean plasma triglyceride level of dams in the HFCS group was $61.2 \pm 9.87 \mathrm{mg} / \mathrm{dl}$ and in the dams of the sucrose group was $53.5 \pm 4.06 \mathrm{mg} / \mathrm{dl}(\mathrm{P}=0.05)$. Mean plasma triglyceride level was $67.6 \pm 6.29$ $\mathrm{mg} / \mathrm{dl}$ of pups in the HFCS group whereas $45.1 \pm 8.35 \mathrm{mg} / \mathrm{dl}$ of pups in the sucrose group $(\mathrm{P}=0.05)$.

Conclusion: Large amounts of maternal fructose intake, during preconception, throughout pregnancy and lactation periods, increase both plasma glucose and triglyceride levels in dams and pups compared with sucrose. Consequently, high amount of fructose, taken with processed foods, in maternal diet may increase the risk for chronic diseases (cardiovascular diseases, type II diabetes mellitus) for both mothers and their babies.

Supported by Hacettepe University (THD-2015-5528). 


\title{
P40
}

\section{Genetic Variants Influence HDL Subclass Distribution in Response to Dietary Cholesterol and Plant Sterol Consumption in a Hutterite Founder Population}

\author{
Peter A.S. Alphonse ${ }^{1,2}$, Peter K. Eck ${ }^{1}$, Peter J.H. Jones ${ }^{2,3}$
}

${ }^{1}$ Department of Human Nutritional Sciences, University of Manitoba, Canada; ${ }^{2}$ Richardson Centre for Functional Foods and Nutraceuticals, University of Manitoba, Canada; ${ }^{3}$ Department of Food Science, University of Manitoba, Canada

Background: Determining plasma lipoprotein subclass distribution confers greater predictive power in estimating cardiovascular disease risk in individuals. Dietary cholesterol and plant sterols affect plasma LDL and HDL lipoprotein subclasses. However, very limited information exists on the influence of common gene polymorphisms on the variability of LDL and HDL subclass distribution in response to dietary sterols.

Objective: Using a candidate gene approach, we investigated the modulatory effects of common genetic variants on plasma HDL and LDL subclass distribution in response to dietary cholesterol and plant sterol consumption in a Hutterite founder population.

Design: A double-blind, randomized, crossover, placebo-controlled study with three dietary intervention phases of 4 weeks each, separated by a 4 -week washout was conducted in 49 healthy Hutterite individuals residing in Manitoba. Participants consumed daily either $2 \mathrm{~g}$ of plant sterols or $600 \mathrm{mg}$ of cholesterol incorporated into milkshakes for 4 weeks during each interventional period. Plasma LDL and HDL subclasses were fractionated and quantified and all participants were genotyped for 38 candidate SNPs across 25 genes involved in lipoprotein and cholesterol metabolism. This trial was registered at clinicaltrials.gov (NCT01825668).

Results: Dietary cholesterol and plant sterols did not alter plasma LDL-C or LDL-subclass distribution. Dietary cholesterol consumption induced an increase in HDL-C $(0.08 \pm 0.03 \mathrm{mmol} / \mathrm{L}, \mathrm{p}=0.0216)$ and large HDL subclasses $(0.12 \pm 0.04$ $\mathrm{mmol} / \mathrm{L}, \mathrm{p}=0.0167$ ) compared to the control, whereas plant sterol consumption did not affect HDL-C or HDL subclass distribution. Among the candidate SNPs, ABCA1 rs2066714 ( $\mathrm{p}=0.0112$ ), SCD rs2234970 ( $\mathrm{p}=0.0024)$, SREBF1 rs2297508 ( $\mathrm{p}=0.0458)$, LIPF $r$ s814628 ( $\mathrm{p}=0.0410)$ modified HDL-2 subclass responsiveness; and ABCA1 rs2066714 ( $\mathrm{p}=0.0176)$, LIPC $r s 6083(\mathrm{p}=0.0273$ ) modified HDL-3 subclass responsiveness to dietary cholesterol consumption.

Conclusion: Results suggest that common genetic variants influence dietary cholesterol consumption induced responsiveness of plasma HDL-C and HDL subclass distribution in healthy Hutterite individuals. 


\section{P41 \\ Fruit Consumption May Protect Against the Development of Intestinal Inflammation via Modification of Microbial Composition}

Lihi Godny 1,2, Nitsan Maharshak 1,2, Lior Yahav ${ }^{1,2}$, Naomi Fliss-Isakov'2, Uri Gophna ${ }^{3}$, Hagit Tulchinsky 2,4, Iris Dotan ${ }^{1,2}$

${ }^{1}$ IBD Center, Department of Gastroenterology and Liver Disease, Tel Aviv Sourasky Medical Center, Israel; ${ }^{2}$ Sackler Faculty of Medicine, Tel Aviv University, Israel; ${ }^{3}$ Department of Molecular Microbiology and Biotechnology, George S. Wise Faculty of Life Sciences, Tel Aviv University, Israel; ${ }^{4}$ Proctology Unit, Department of Surgery, Tel Aviv Sourasky Medical Center, Israel

Background: Patients with ulcerative colitis (UC) undergoing proctocolectomy with ileal pouch-anal anastomosis commonly develop pouchitis. As pouchitis is inflammation developing in a previously normal small bowel we hypothesize that it may represent the small intestinal inflammation characterizing Crohn's disease (CD). CD pathogenesis involves environmental factors. We thus asked whether diet and the microbiome had a role in pouch inflammation.

Methods: Patients recruited at our Comprehensive Pouch Clinic were prospectively followed-up. Pouch behavior was defined as normal pouch (NP) or pouchitis. All patients completed Food Frequency Questionnaires. Fecal samples were analyzed for microbial composition.

Results: A total of 172 patients ( 89 [52\%] females, age $44.9 \pm 14$ years, mean time since ileostomy closure 9.1 [range $0-30.4$ ] years) were recruited. At the beginning of follow up, 39 (22.6\%) patients had NP. Within 1 year of follow up, 5 $(12.8 \%)$ of these developed pouchitis. Higher (1.45 servings/day), compared to lower fruit consumption was associated with significantly less development of pouchitis ( $3.8 \%$ vs. $30.8 \%$, respectively, log rank test, $\mathrm{p}=0.03$ ). Fecal microbial analysis was performed in 81 patients (NP [ $=22]$, pouchitis [ $=53]$, familial adenomatous polyposis $[\mathrm{n}=7])$. Fruit consumption correlated with microbial diversity $(r=0.37, p=0.001)$, and with the abundance of several microbial groups. After adjustment for pouch behavior and use of antibiotics, fruit consumption remained positively correlated with Faecalibacterium $(r=0.27, p=$ 0.01), Lachnospira $(\mathrm{r}=0.31, \mathrm{p}=0.005)$ and two un-annotated genera from the Lachnospiraceae and Ruminococcaceae families $(\mathrm{r}=0.24 \mathrm{p}=0.03 ; \mathrm{r}=0.28, \mathrm{p}=$ 0.01 , respectively). Significant decrease in fruit consumption was noticed in 10 patients who developed active disease over time $(\Delta=-1.4 \pm 1.7 \mathrm{~s} / \mathrm{d}, \mathrm{p}=0.02)$. Corresponding decrease in microbial diversity was noticed as well $(\Delta=-0.6 \pm 1.2$, $\mathrm{p}=0.17$.

Conclusions: Fruit consumption by pouch patients modified microbial composition. Significant decrease in fruit consumption was associated with the development of active disease and with a decreased microbial diversity. Therefore, dietary intervention may contribute to prevention of intestinal inflammation. 


\title{
P42
}

\section{The Effects of Diet Contamination on Wide Genome Expression in Duodenum of Growing Pigs}

\author{
Gina Cecilia Pistol', Cornelia Braicu²,3, Mihail Alexandru Gras, \\ Daniela Eliza Marin ${ }^{1}$, Ionelia Taranu ${ }^{1}$ \\ ${ }^{1}$ Laboratory of Animal Biology, National Institute for Research and \\ Development for Biology and Animal Nutrition, Balotesti, Ilfov, Romania; \\ ${ }^{2}$ Oncologic Institute, Prof. Dr. I. Chiricuta, Cluj Napoca, Romania; \\ ${ }^{3}$ Research Center for Functional Genomics, Biomedicine and Translational \\ Medicine, 'Iuliu Hatieganu' University of Medicine and Pharmacy, Cluj- \\ Napoca, Romania
}

Cereals and cereal products are significant and important animal and human feed and food resources worldwide. Among the most frequent contaminants of cereal commodities are mycotoxin-producing moulds and pathogenic microorganisms, pesticides, etc. Zearalenone (ZEN) is a mycotoxin produced by Fusarium species of fungi. The aim of the present study was to assess whether the in vivo exposure of growing pigs to $140 \mathrm{ppb}$ ZEA contaminated diet and other contaminants generates changes in wide genome expression at duodenal level using a DNA microarray. The gastrointestinal tract, especially the small intestine, is primarily responsible for digestion and absorption of dietary nutrients, as well as a barrier against potential pathogens and toxins and a key organ for the local immune response. The microarray data identified 350 genes significantly $(\mathrm{P}=$ 0.005) differentiated expressed in duodenum of contaminated pigs compared to control; of these 59.7\% (209 genes) were down-regulated and 40.3\% (141 genes) up-regulated. The extrapolation of microarray data to human genes annotation leaded to identification of important genes which were modulated by contamination exposure. For example, the experimental contamination was associated with significant $(\mathrm{P}=0.005)$ down-regulation of genes coding for important immune mediators as chemokines (CCL8, CXCL14) and human leukocyte antigen system (HLA-A, HLA-DPQ). Also, diet contamination leaded to the up-regulation of genes required for the $S$ phase entry of the cell cycle (CDC123), ribosomal proteins (RPL38) and immune mediators (CCL11). In summary, these results suggest that exposure of growing pigs to zearalenone and other contaminants occurred in cereal feed produced significant changes in the wide genome expression of duodenum as a first route for oral intoxication. 


\section{P43}

\section{Omega-3 Deficiency Impairs Honey Bee Learning}

Yael Arien ${ }^{1}$, Arnon Dag ${ }^{2}$, Shlomi Zarchin ${ }^{1}$, Tania Masci ${ }^{1}$, Sharoni Shafir ${ }^{1}$

${ }^{1}$ B.Triwaks Bee Research Center, Department of Entomology, Robert $\mathrm{H}$. Smith Faculty of Agriculture, Food \& Environment, The Hebrew University of Jerusalem, Rehovot, Israel; ${ }^{2}$ Gilat Research Center, Institute of Plant Sciences, Agricultural Research Organization, Israel

Pollen is essential component in honey bee nutrition. It is the main source for protein but also provides fatty acids, minerals and vitamins. Fatty acids content and composition in pollen varies between different types of plants. In modern agriculture bees are placed in large areas of monoculture (single-species crops), where bees forage on an unbalanced pollen diet that could lead to malnutrition.

In mammals, the importance of essential omega-3 fatty acids is well known, and deficiency of omega-3 fatty acids, mainly long-chain polyunsaturated fatty acids (PUFAs), is associated with several mental and cognitive disorders. Insects, on the other hand, have very low levels of long-chain PUFAs and the nutritional effect of deficiency in omega-3 has not yet been studied. In this research we investigated for the first time the importance of essential omega-3 fatty acids in honey bee nutrition.

We performed experiments examining the effect of omega-3 deficiency on olfactory and tactile associative learning ability via Proboscis Extension Response assays. Bees were fed artificial diets enriched with vegetable oils that were rich or poor in omega-3, and mixed bee-collected pollen pellets, as omega-3-rich diet, or Eucalyptus pollen pellets, which are poor in omega-3.

We show a physiological effect; there was a decrease in the size of hyphopharyngeal glands (produce queen and worker jelly) of bees fed omega-3 deficient diets. Omega-3 levels in bee's body were greatly affected by diets, and omega-3 levels in bee's brain were only slightly affected by diet. We also found a decrease in the learning ability of bees that were fed each of the low omega-3 diets, both artificial and pollen-pellet based diets. These findings suggest that, similar to mammals, omega-3 fatty acids have a crucial role for cognitive function of honey bees; this is shown here for the first time in invertebrates. 


\section{P44}

Factors Influencing the Formation of Nisin and Its Isolation from Whey and the Use of Antimicrobial Effects for Food, Cosmetic and Medical Applications

Alexandra Salakova ${ }^{1}$, Michael Binder ${ }^{1}$, Antonín Nehyba ${ }^{1}$, Vladimír Sedlař́k ${ }^{2}$, Zuzana Kolářová Rašková ${ }^{2}$, Jan Drbohlav ${ }^{1}$, Petr Roubal $^{1}$

${ }^{1}$ Research, Vyzkumny Ustav Mlekarensky, Czech Republic; ${ }^{2}$ Centrum

Polymernich Systemu, Univerzita Tomase Bati ve Zline, Univerzitni institut, Czech Republic

The agar diffusion method on the BHI agar with use of the strain indicator Micrococcus luteus was selected for monitoring of nisin produce by 3 strains of Lactococcus lactis and the method conditions were optimized. The tested strains were inoculated to milk and sweet whey. The strain CCDM 731 after 16 hours appeared significantly the fastest coagulation. This strain was further cultured in sweet whey stationary or with shaking for $0-42$ hours. The aeration of the substrate did not affect the formation of nisin, the maximum concentration was detected after 4-8 hours. Testing of various suplements in the whey to increase nisin produce proved the best result use of $0.1 \%$ Tween 80 .

Antimicrobial proteins of whey were isolated by means of ion exchange chromatography. The polyacrylamide gel electrophoresis was used for the protein identification. The synergy in antimicrobial action of lactoferrin and nisin was observed. 


\section{P45}

A Methyl Balanced Diet Can Prevent Prenatal CRF Triggered Predisposition to Binge Eating

Mariana Schroeder $^{1,2}$, Tamar Polacheck ${ }^{1,2}$, Yonat Drori $^{1,2}$, Maya Lebow ${ }^{1,2}$, Shifra Ben-Dor ${ }^{3}$, Alon Chen ${ }^{1,2}$

${ }^{1}$ Department of Stress Neurobiology and Neurogenetics, Max-Planck Institute of Psychiatry, Germany; ${ }^{2}$ Department of Neurobiology,

Weizmann Institute of Science, Israel; ${ }^{3}$ Bioinformatics and Biological

Computing Unit, Weizmann Institute of Science, Israel

Binge eating is a common aberrant form of eating behavior, characterized by overconsumption of food in a brief period of time. Recurrent episodes of binge eating constitute the binge eating disorder (BED), which mostly affects females and is associated with early life adversities. Here, we show that overexpression of maternal corticotropin-releasing factor in late gestation predisposes adolescent female offspring to binge eating-like behavior, that coincides with epigenetically mediated changes in the hypothalamus at multiple levels. We demonstrate that gestational programming per se will not lead to binge eating but only when challenged during adolescence, pre-existing alterations due to prenatal programming are revealed. Moreover, exposing the offspring to a balanced methyl donor diet during adolescence can prevent this predisposition from being triggered. We provide empirical mechanistic evidence for long-term epigenetic abnormalities stemming from a stressful prenatal environment, in predisposing female offspring to BED as well as a potential non-invasive prevention strategy. 


\section{P46}

A Randomized Controlled Trial of a Gluten-Free Weight Loss Diet in HLA DQ2 or D8 Positive Subjects

Maria Vranceanu ${ }^{3}$, David de Lorenzo ${ }^{1,2}$, Keith A. Grimaldi ${ }^{3}$

${ }^{1}$ Departament de Ciencies Experimentals i de la Salut, Universitat Pompeu Fabra, CEXS-UPF-PRBB, Barcelona, Spain; ${ }^{2}$ Centro de Estudios en Genomica y Nutricion-CESGEN, Parc Cientific i Tecnolgic Agroalimentari de Lleida-PCiTAL, Spain; ${ }^{3}$ Nutrigenetics, Eurogenetica, UK

Introduction and Objectives: Despite the health claims for gluten-free eating, there are no published reports showing that a gluten-free diet produces weight loss in persons without celiac disease. In fact, there are data to suggest that gluten itself may provide some health benefits, and that gluten avoidance may not be justified for otherwise healthy individuals. Our primary purpose is to try to provide experimental evidence for the effect on weight loss of a gluten-free diet, and analyze the potential interactions between genetic factors affecting gluten sensitivity (located at the HLA-DQ gene) and gender.

HLA and Gluten

- Approximately 99\% of people with celiac disease have DQ2 isoform (mainly subtype 2.5, a haplotype with two adjacent genes encoding the two subunits and $\beta 2 \alpha 5$ ) or DQ8 (subtype 8.1, a haplotype of two genes with variants $\alpha 3-\beta 8)$.

- The reason why these genes result in increased risk of celiac disease is that the receptors formed by them gliadin peptides (a component of gluten) stronger than other forms presenter bind antigen receptor.

Methodology: DQ2.2, DQ2.5, DQ7 and DQ8 serotypes were estimated using the 7-SNP panel described by Monsuur et al (PLoS One 2008;3:e2270) with the Eurogenetica Nutrigene+ test. We tested 215 patients. 221 patients were overweight or obese and 69 patients had at least one allele for DQ2 or DQ8. 64 were overweight and were randomly divided into two groups, both were prescribed a $1400 \mathrm{kcal} /$ day diet and group a gluten-free diet.

Results: The gluten-free group showed a significant weight loss compared to the control group at three and six months (3 m: $14 \%$ vs. $8 \%$ and $6 \mathrm{~m}$ : $25 \%$ vs. $14 \%)$. A gluten elimination diet in patients with at least one risk allele for gluten sensitivity correlated to a significantly more weight loss in patients with weight problems than a standard diet. 


\section{P47}

\section{Effect of 3-Hydroxy-3-Methylbutyrate on Protein Metabolism in Whole Body and in Selected Tissues of Laboratory Rat}

Milan Holecek, Miroslav Kovarik, Melita Vodenicarovova, Ludek Sispera

Department of Physiology, Charles University in Prague, Faculty of Medicine in Hradec Kralove, Czech Republic

3-hydroxy-3-methylbutyrate (HMB) is a leucine metabolite with protein anabolic action which may be employed in treatment of proteocatabolic illness and in endurance training. We examined the effects of exogenous HMB on protein synthesis and proteolysis in whole body and selected tissues of laboratory rat.

Rats were administered by HMB at a dose of $0.1 \mathrm{~g} / \mathrm{kg}$ b.w. (s.c. and i.v., 1:1) or by saline (control). The parameters of whole-body protein metabolism were evaluated 24 hours later using L-[1-14C]leucine and L-[3,4,5-3H]phenylalanine. Changes in proteasome dependent proteolysis and protein synthesis in selected tissues were determined according the chymotrypsin-like activity and labeled leucine and phenylalanine incorporation into the protein. The results were analyzed using Mann-Whitney test.

A decrease in whole-body proteolysis and protein synthesis was observed in HMB treated rats. Proteasome-dependent proteolysis decreased significantly in skeletal muscle, changes in heart, liver, jejunum, colon, kidney, and spleen were insignificant. Decrease in protein synthesis was observed in the heart, colon, kidney, and spleen, while an increase was observed in the liver.

We conclude that protein anabolic effect of HMB in skeletal muscle is related to inhibition of proteolysis in proteasome. Alterations in protein synthesis in visceral tissues may affect several important functions and the metabolic status of the whole body.

Supported by PRVOUK P37/02. 


\section{P48}

N-Acetyl-Cysteine (NAC) Supplementation Improves Insulin Sensitivity: A Personalized Recommendation Is Required

Alona Falach ${ }^{1,2}$, Hava Rozenfeld ${ }^{1,2}$, Lital Argaev-Frenkel', Sanford R. Sampson ${ }^{3}$, Moria Chetboun ${ }^{1,2}$, Tovit Rosenzweig ${ }^{1}$

${ }^{1}$ Departments of Molecular Biology and Nutritional Studies, Ariel University, Israel; ${ }^{2}$ Faculty of Life Sciences, Bar-Ilan University, Israel; ${ }^{3}$ Department of Molecular Cell Biology, Weizmann Institute of Science, Israel

Introduction: Meta analyses conclude that antioxidant (AOX) supplementations have no beneficial effects on the prevalence of type 2 diabetes (T2D). These disappointing results conflict with most in-vitro and in-vivo studies showing interference with oxidative stress and benefits of AOXs on insulin sensitivity and $\beta$-cell function. Recently, the role of reactive oxygen species in a large number of physiological functions, including transmission of insulin signaling, was demonstrated. We suggest that while AOXs are beneficial for reduction of oxidative stress, inappropriate intake of AOX, either at lower or higher than the optimal dose, impairs the effectiveness of treatment. The aim of this study is to clarify the dose-response effects of the AOX N-acetyl-cysteine supplementation on the progression of T2D.

Methods: Experiments were conducted on KK-Ay mice, given NAC at different concentrations (200-1,800 mg/kg/day) for 6 weeks. Glucose and insulin tolerance tests were performed and plasma insulin, total antioxidant capacity (TAC) and lipid peroxidation were measured. Insulin signaling pathway was followed in soleus muscle and pancreas was stained by H\&E.

Results: Although lipid peroxidation was reduced and TAC elevated in all concentrations used, glucose intolerance was not corrected in the $200 \mathrm{mg} / \mathrm{kg} /$ day treated mice. While 600, 1200 and $1800 \mathrm{mg} / \mathrm{kg} /$ day NAC were all found to improve glucose tolerance, only the $1200 \mathrm{mg} / \mathrm{kg} /$ day treatment increased insulin sensitivity as indicated by improved insulin tolerance test, reduced plasma insulin and improved transmission of insulin signaling in soleus muscle and liver of treated mice. Islet hypertrophy was also corrected only in the 1200 $\mathrm{mg} / \mathrm{kg} /$ day treated animals.

Conclusion: The inconsistency in the literature regarding AOXs and prevention of diabetes may result from the lack of clear guidelines for effective doses. Thus, there is a need to identify certain biomarkers for the target oxidative state that should be maintained in order to obtain optimal outcomes. 


\section{P49 \\ Insulin Sensitivity Is Improved by the Beduin Medicinal Plant, Sarcopoterium Spinosum}

Konstantin Rozenberg ${ }^{1}$, Nir Skalka 1,2, Sanford R. Sampson ${ }^{3}$, Zohar Kerem ${ }^{2,4}$, Tovit Rosenzweig,

${ }^{1}$ Molecular Biology, Ariel University, Israel; ${ }^{2}$ Diabest Botanical Drugs Ltd, Israel; ${ }^{3}$ Department of Molecular Cell Biology, Weizmann Institute of Science, Israel; ${ }^{4}$ Robert H. Smith Faculty of Agriculture, Food and Environment, Hebrew University, Israel

Introduction: Sarcopoterium spinosum (Thorny burnet) is an abundant plant in Israel, used by Beduin medicinal practitioners for the treatment of diabetes. The aim of this study was to clarify its mechanism of action in the treatment of type 2 diabetes (T2D).

Methods: In-vivo studies were performed on KK-Ay (spontanously developing T2D) and high fat diet-fed mice given the extract for 6 and 9 weeks respectively. Glucose and insulin tolerance tests were performed, and the activation of insulin signaling pathway in soleus muscle and liver was followed. Mechanisms of action were investigated in L6 myotubes and 3T3-L1 adipocytes using Western blot analysis and glucose uptake assay.

Results: S. spinosum extract improved glucose tolerance and insulin sensitivity in treated mice, KK-Ay mice being more sensitive for the treatment. S. spinosum extract increased glucose uptake in 3T3-L1 adipocytes. An additive effect was found between $S$. spinosum and insulin. Using specific inhibitors we showed that while inhibition of PI3K did not affect $S$. spinosum-dependent glucose uptake, inhibition of AKT completely abrogated this effect, suggesting that $S$. spinosum activates AKT via PI3K independent mechanism. This unique mechanism of action was confirmed using Western blot analysis, showing that in contrast to insulin action, neither ser473 nor thr308 was phosphorylated by S. spinosum. However, translocation of PKB from the cytoplasm to the membrane and the nucleus was detected. In addition, events downstream of AKT activationGSK3 $\beta$, FOXO and PRAS40 phosphorylation, and GLUT4 translocation-were increased by the extract. AMPK is not involved in the effects of $S$. spinosum.

Conclusion: We propose that the active ingredients in $S$. spinosum activatePKB by a unique mechanism which is independent of ser473 and thr308 phosphorylation. Identifying the active molecules and clarifying its mechanism of action may lead to the development of new agents for the treatment of insulin resistance. 


\section{P50}

\section{Developing Peptidic Inhibitors for E. Coli Mazf Toxin Protein}

\section{Carmit Ginesin Tzela, Zvi Hayouka}

Institute of Biochemistry, Food Science and Nutrition, The Robert

$\mathrm{H}$. Smith Faculty of Agriculture, Food and Environment, The Hebrew

University of Jerusalem, Israel

One of the challenges in antibiotic treatment is the re-infection that may be caused by persister cells. These sub-population bacterial cells enter a state of dormancy and therefore are not affected by antibiotic treatment. The development of persister cells seems to be induced by bacterial Toxin-Antitoxin (TA) systems. Reducing or preventing the presence of persister cells may abrogate re-infection after antibiotic treatment.

TA systems are composed from toxin and antitoxin proteins. In the Escherichia coli mazEF TA system, the toxin mazF is an endoribonuclease and the antitoxin mazE binds mazF in a complex that prevents mazF catalytic activity. The antitoxin is less stable, and when it is being degraded the toxin is released and may induce bacterial cell death.

Our goal is to study the Escherichia coli mazEF TA system at the molecular level using peptides based tools. In the current research we designed and synthesized peptides based on structural and biochemical findings. Both toxin and antitoxin proteins were expressed and purified in high yield and purity. mazF endoribonuclease catalytic activity was measured using a fluorometric assay (FRET). We characterized the effect of the designed peptides on mazF catalytic activity. We have found a peptide that inhibits mazF activity at low micromolar concentrations. We are currently studying the effect of our peptides on E. coli cells in vivo to better understand its biological role. In the future our new compounds may be used as a combined treatment with antibiotics to prevent re-infection. 


\section{P51}

\section{Consumption of Soy-Based Infant Formula Is Not Associated with Early Onset of Pubertal Signs}

Tali Sinai ${ }^{1}$, Shely Ben-Avraham², Inbal Guelmann-Mizrahi', Michael R. Goldberg ${ }^{3,4}$, Larisa Naugolni', Galia Askapa², Yitzhak Katz ${ }^{3,4}$, Marianna Rachmiel ${ }^{4}$

${ }^{1}$ School of Nutritional Sciences, The Hebrew University of Jerusalem, Israel; ${ }^{2}$ Pediatric Endocrinology Unit, Assaf-Harofeh Medical Center, Israel; ${ }^{3}$ Allergy and Immunology Institute, Assaf-Harofeh Medical Center, Israel;

${ }^{4}$ Sackler Faculty of Medicine, Tel Aviv University, Israel

Background: Earlier presentation of puberty is suggested to be associated with early phytoestrogens consumption.

The objective of this study was to examine prospectively the association between consumption of soy-based foods in early infancy and the incidence of early pubertal signs in school-aged children.

Methods: The study cohort was pooled from infants followed prospectively until age 3 for the development of IgE-mediated cow's milk allergy. Milk allergic patients who consumed only soy based formula and foods during infancy and early childhood (soygroup, $\mathrm{n}=30$ ) were compared to a control group randomized from the healthy infants with a non-soy based intake $(n=60)$. Study participants were reevaluated at school age for physical activity, current food habits (collected from 3 days food diaries), and growth and puberty parameters on physical examination.

Results: The study population $(\mathrm{n}=90)$ consisted of 46 males and 44 females, (ages $8.9 \pm 0.6$ years versus $8.4 \pm 0.5$ years, mean $\pm S D$, respectively, $p=0.0007$ ) Timing of pubertal signs (adrenarche and gonadarche) was similar in both groups. Four from the soy group (13.8\%) and 8 from the non-soy group (13.3\%) had early pubertal onset. The daily consumption of energy, carbohydrates, fat and protein nutrients were similar between the groups. Similar height and BMI $\mathrm{z}$ scores were noted between the soy and the non-soy fed groups $(-0.16 \pm 1.02$, $-0.18 \pm 0.97, \mathrm{p}=0.92$, versus $0.64 \pm 1.03,0.56 \pm 1.02, \mathrm{p}=0.72$, respectively)

Conclusions: This is the first prospective, long term follow up study of a pediatric cohort, demonstrating there is no association between infantile soy based formula consumption and early signs of puberty. 59 . 


\section{P52}

\section{Improving Agriculture Products Quality and Safety by Novel} Antimicrobial Random Peptide Mixtures

Tal Stern Zvi Hayouka

Institute of Biochemistry, Food Science and Nutrition, Faculty of Agriculture, Food and Environment, The Hebrew University of Jerusalem, Israel

Microorganisms have a major role in food spoilage and safety. Improving existing ways to deal with microorganism in food and exploring the typical bacteria of each food product is essential for the development of effective antimicrobial tools to tackle them. In our research, we focus on milk as a food model that it shelf life depends on microorganism activity as it contains a variety of nutrients provide the bacteria an ideal growth environment and for human a nutritional value product. We used MALDI-TOF mass spectrometry method for identification of isolated bacteria from pasteurized milk that was spoiled in different conditions. MALDI-TOF mass spectrometry is a novel, rapid and simple method for bacteria identification based on unique fingerprint of specific bacteria. Novel antimicrobial random peptide mixtures that we have developed recently were studied as active agents towards the isolated spoilage milk bacteria. Random peptides mixture material was generated using solid-phase peptide synthesis method in unconventional way, instead of using one pure amino acid at each coupling step we used a mixture of two amino acids in a defined proportion hydrophobic and cationic residues. Each mixture was random in terms of sequence, but highly controlled in terms of chain length and stereochemistry. We found that the random peptide mixtures have strong and broad antimicrobial activity towards most of the milk spoilage bacteria that were isolated during this study. According to these findings, we will develop milk bioactive food packaging using random peptide mixture to increase milk safety and shelf life. 


\title{
P53 \\ Interaction Effect of Genetic Variants in GC and DHCR7 and the Vitamin D Status on Blood Pressure and the Risk of Gestational Hypertension in Pregnant Women
}

\author{
Jayong Chung ${ }^{1}$, Hyo-Suk Lee ${ }^{1}$, Soo-Kyung Chun ${ }^{1}$, Hyojee Joung ${ }^{2}$, \\ Mun-Young Kim ${ }^{3}$ \\ ${ }^{1}$ Department of Food and Nutrition, Kyung Hee University, South Korea; \\ ${ }^{2}$ Graduate School of Public Health, Seoul National University, South \\ Korea; ${ }^{3}$ Department of Obstetrics and Gynecology, Cheil General Hospital, \\ South Korea
}

Gestational hypertension $(\mathrm{GH})$ is one of the most common diseases resulting in maternal and fetal complications in pregnancy. Previous studies have reported the associations between vitamin D (VD) status and blood pressure. In this study, we investigated the influence of single nucleotide polymorphisms (SNP) in genes associated with vitamin D metabolism such as vitamin D binding protein (GC) and 7-dehydrocholesterol reductase (DHCR7) and VD status on blood pressure (BP) in pregnant women. We recruited 399 pregnant women at early pregnancy and measured their serum 25-hydroxy-VD levels by LC-MS/MS method. BP was measured at the mean of 35 gestational weeks. 35\% of the pregnant women were VD deficient [serum 25-hydroxy-VD $20 \mathrm{ng} / \mathrm{ml}$ ]. Compared to VD sufficient group, VD deficient group had significantly higher levels of SBP, DBP, PP and MAP (P = 0.05). Also, we found that the association between SNPs in the GC and DHCR7 gene and blood pressure were differed by the VD status. GC SNP rs7041 significantly modified the SBP, DBP, and MAP only in the VD deficient group, and no such relationship was found in the VD sufficient group ( $\mathrm{p}$ for interaction 0.05). Similarly, DHCR7 SNP rs3829251 significantly modified SBP or MAP in the VD deficient group but not in the VD sufficient group ( $\mathrm{p}$ for interaction 0.05). Further, the risk of GH were significantly increased by the VD deficiency (OR $=10.33,95 \%$ $\mathrm{CI}=1.31-81.44$ ) only in subjects with rs7041 TT genotypes but not in subjects with rs7041 GT or GG genotypes (OR $=0.84,95 \% \mathrm{CI}=0.27-2.61)$. In summary, our findings suggest that the interaction between GC and DHCR7 genetic variations and VD status may modify the risk of developing GH in pregnant women. [Supported by the National Research Foundation of Korean Government (2013R1A1A2007613)]. 


\section{Selected Abstracts}

\section{4}

\section{Genetic Improvement of Sesame Seeds Nutrient Content} and Bioavailability

Naama Teboul', Shimrit Bar-El ${ }^{2}$, Yaron Gadri', Itamar Vilan', Zipi Berkovich', Ram Reifen ${ }^{2}$, Zvi Peleg $^{1}$

${ }^{1}$ The Robert H. Smith Institute for Plant Sciences and Genetics in Agriculture, The Hebrew University of Jerusalem, Israel; ${ }^{2}$ Institute of Biochemistry, Food Science and Nutrition, The Hebrew University of Jerusalem, Israel

Sesame (Sesamum indicum spp.) is an important oil-crop worldwide, which known for its high nutritional values. Sesame seeds are used for cooking, or crushed for producing high-grade edible oil or oily paste (Tahini) and sweets. Despite the continuous increase in demand for sesame as a health food, sesame consider to be an orphan crop and it has been subjected to only limited research. Micronutrient malnutrition afflicts over two billion people worldwide and prevalent mostly in developing countries where the diet lacks diversity and based mainly on a single staple food. Therefore, enhancement of grain nutrients (biofortification), either agronomically or genetically, is considered the most promising and cost-effective approach to alleviate malnutrition and related health problems. The aim of the current study is to dissect the genetic, physiological and biochemistry factors affecting seed nutrient content. A large collection of worldwide sesame genotypes was characterized for agronomically important traits and seed mineral-nutrients content. A wide genetic diversity in morphological and micronutrients content was found among genotypes. Our results revealed a genetic potential for improving micronutrient content and especially iron in sesame. In order to measure iron bioavailability in different sesame accessions, we use static in-vitro digestibility model in which gastric and small intestine digestion is mimicked in two consecutive steps, allowing the analysis of soluble as well as insoluble iron content. A mapping population derived from a cross between two contrasting sesame genotypes, was used for genetic dissection of seed mineral-nutrient. Using genotyping by sequencing, we were able to identify and characterize several quantitative trait loci (QTLs) conferring high seed nutrient contents. Revealing the molecular basis of nutrient accumulation in sesame will contribute to the development of toolkits for high yielding sesame varieties with enhanced nutritional values. Our results demonstrate the potential of using sesame as a model for biofortification study. 


\section{Traits and Properties Control of Bulk Tank Milk}

Gil Katz ${ }^{1}$, Dror Bezman ${ }^{1}$, Liubov Kuzin1, Uzi Merin'1, Gabriel Leitner ${ }^{2}$

${ }^{1} R \& D$, Afimilk, Israel; ${ }^{2}$ National Mastitis Reference Center, Kimron

Veterinary Institute, Israel

Raw milk chemical and physical properties are governed by many factors such as genetics, diet, lactation stage, parity, environment and health. These factors vary between cows, during lactation, between milking sessions but most of all during a single milking of an individual cow. Modern farms exercise decision making using data collected by sensors installed in parlor or on individual cows. Employing high-resolution data of milk traits available in real time allows channeling milk during the milking session to different bulk tanks according to predetermined required traits and quantities. Cheese manufacturing efficiency is determined mainly by the level of milk constituents and its coagulation properties, i.e., rennet clotting time (RCT) and curd firmness (CF). Real time milk routing conducted on a farm milking 516 Israeli Holstein cows with a daily average of $37.5 \mathrm{~kg}$ milk. Cheese production experiments were conducted using milk separated in real time using the Afilab $^{\circledR}$ milk spectrometer. The spectrometer measures milk components at each stall: fat, protein, lactose, casein, fatty acids and coagulation potential, i.e, Afi-Cf. Properties and components as well as the variations during the milking session are detected in real time. The system is employed for sorting milk in real time into two Tanks: A) for cheese production (CM), or B) for fluid milk products (FM). Cheese was prepared from milk acquired at varying separation ratios of the expected farm daily yield. Milk was preprocessed correcting protein/fat ratio to 1.0. Cheese weight increased along the cutoff level with no difference in moisture, while dry matter increased. On the farm, optimizing milk for cheese making results in an increase of $10-15 \%$ in cheese yield. The same resources extent can yield more profits by obtaining sustainability, supporting milk plants interest for higher cheese yield and herdsmen for receiving higher profits for the same amount of raw milk. 
Changes in Cardiotrophin-1 Serum and PBMC Expression Levels after a Weight Loss Program in Obese Children

\author{
Amelia Marti ${ }^{1,4,5}$, Lydia Morell-Azanza ${ }^{1,5}$, Tara Rendo-Urteaga ${ }^{1}$, \\ Sonia Garcia-Calzon ${ }^{1}$, Maria Chueca ${ }^{2,5}$, Maria Jesus Moreno-Aliaga 1,4,5, \\ J. Alfredo Martinez ${ }^{1,4,5}$, Cristina Azcona-SanJulian ${ }^{3,5}$ \\ ${ }^{1}$ Nutrition, Food Sciences and Physiology, University of Navarra, Spain; \\ ${ }^{2}$ Pediatric Endocrinology Unit, Complejo Hospitalario de Navarra, Spain; \\ ${ }^{3}$ Department of Pediatrics, Clinica Universidad de Navarra, Spain; ${ }^{4} \mathrm{CIBER}$ \\ Fisiopatologa de la Obesidad y Nutricion (CIBERobn), Instituto de Salud \\ Carlos III, Spain; ${ }^{5}$ Navarra Institute for Health Research, Pamplona, Spain
}

Introduction: Cardiotrophin-1 (CT-1) belongs to the interleukin (IL)-6 family of cytokines and high levels of CT-1 levels are linked to obesity and metabolic syndrome (Lopez Yoldi 2015). A successful weight loss (WL) program is able to lower cardiovascular risk factors. Interestingly, in obese children we have shown a reduction in serum CT-1 levels after a WL program being the CT-1 decrease strongly associated with a reduction in cholesterol levels and metabolic syndrome risk factors (Rendo Urteaga et al. 2013). Here, we further characterize the association between changes CT-1 gene expression and serum levels and their relationship with IL-6 levels after a 10-week WL program in obese children.

Subjects and Methods: Twenty-three obese children (mean age 12.3 years; $48 \%$ males) undergoing a 10-week weight loss program were enrolled. CT-1 and IL-6 gene expression analysis was carried out by RT-PCR in peripheral blood mononuclear cell (PBMC). Serum cytokines levels were also measured.

Results: In obese children CT-1 gene expression decreased significantly after the 10 -week WL intervention ( $p=0.005)$. Interestingly, changes in serum CT-1 levels were also significantly correlated with changes in CT-1 gene expression $(r=0.451, p=0.031)$. Moreover, changes in CT-1 gene expression were also correlated with changes in IL-6 gene expression $(r=0.646, p=0.001)$.

Conclusion: We show for the first time that in obese children changes in serum CT-1 levels does mirror changes in CT-1 gene expression in PBMC after a WL program. 
Carbohydrate Intake and Physical Activity Levels Modify the Association between FTO Gene Variants and Obesity and Type 2 Diabetes: First Nutrigenetics Study in an Asian Indian Population

Vimal Karani ${ }^{1}$, Dhanasekaran Bodhini ${ }^{2}$, N. Lakshmipriya ${ }^{2}$, K. Ramya ${ }^{2}$, Anjana R. Mohan ${ }^{2,5}$, Sudha Vasudevan ${ }^{3}$, Sanjay Kinra ${ }^{4}$, Julie Lovegrove ${ }^{1}$, Mohan Viswanathan ${ }^{2,5}$, Radha Venkatesan ${ }^{2}$

${ }^{1}$ Food and Nutritional Sciences, Hugh Sinclair Unit of Human Nutrition, UK; ${ }^{2}$ Department of Molecular Genetics, Madras Diabetes Research Foundation, Chennai, India; ${ }^{3}$ Department of Foods, Nutrition and Dietetics Research, Madras Diabetes Research Foundation, Chennai, India; ${ }^{4}$ Department of Non-Communicable Disease Epidemiology, London School of Hygiene \& Tropical Medicine, London, UK; ${ }^{5} \mathrm{WHO}$ Collaborating Centre for Non-Communicable Diseases Prevention and Control, Dr. Mohan's Diabetes Specialties Centre, Chennai, India

Lifestyle factors such as diet and physical activity have been shown to modify the association between fat mass and obesity-associated (FTO) gene variants and metabolic traits in several populations; however, there are no genelifestyle interaction studies, to date, among Asian Indians living in India. In this study, we examined whether dietary factors and physical activity modified the association between two FTO single nucleotide polymorphisms (SNPs) and obesity traits and type 2 diabetes (T2D) in 734 unrelated T2D and 884 normal glucose-tolerant (NGT) participants randomly selected from the Chennai Urban Rural Epidemiology Study (CURES). Interaction analyses were performed by including the interaction terms in the linear/logistic regression model. There was a significant interaction between SNP rs8050136 and carbohydrate energy percentage $(\mathrm{P}$ interaction $=0.04)$, where the ' $\mathrm{A}$ ' allele carriers had 2.46 times increased risk of obesity than those with 'CC' genotype $\left(\mathrm{P}=3.0 \times 10^{-5}\right)$ among individuals in the highest tertile of carbohydrate energy percentage. A significant interaction was also observed between SNP rs11076023 and dietary fibre intake ( $\mathrm{P}$ interaction $=0.0008$ ), where individuals with AA genotype who are in the $3 \mathrm{rd}$ tertile of dietary fibre intake had $1.62 \mathrm{~cm}$ lower waist circumference than those with ' $\mathrm{T}$ ' allele carriers $(\mathrm{P}=0.02)$. Furthermore, among those who were physically inactive, the 'A' allele carriers of the SNP rs8050136 had 1.89 times increased risk of obesity than those with ' $C C^{\prime}$ ' genotype $\left(\mathrm{P}=4.0 \times 10^{-5}\right)$. In conclusion, our findings suggest that the association between FTO SNPs and obesity might be influenced by high carbohydrate and dietary fibre intake and physical inactivity in this Asian Indian population. Further understanding of how FTO gene influences obesity and T2D through dietary and exercise interventions is warranted to advance the development of behavioral intervention and personalised lifestyle strategies, which could reduce the risk of metabolic diseases in this Asian Indian population. 
10th Congress of the International Society of Nutrigenetics/Nutrigenomics (ISNN)

\section{Author Index}

Ahn-Jarvis, J. P34

Alphonse, P.A.S. P40

Argaev-Frenkel, L. 010, P48

Argov-Argaman, N. P25

Arien, Y. P43

Arós, F. P20

Askapa, G. P51

Azcona-SanJulian, C. 56

Bar-El, S. 54

Ben-Avraham, S. P51

Ben-Dor, S. P45

Ben-Ishay, N. P23

Berkovich, Z. 54

Bernstein, S. 012

Beta, T. 011

Bezman, D. 55

Binder, M. P44

Bodhini, D. 57

Bordoni, L. 014, P16

Borel, P. 09

Bott, R. 09

Braicu, C. P35, P36, P42

Calabriso, N. P29, P30, P31

Carluccio, M.A. P29, P30, P31

Chen, A. P45

Chen, J. 06

Chertok, I. P25

Chetboun, M. P48

Choi, B.-H. P15

Chueca, M. 56

Chun, S.-K. P53

Chung, J. P53

Clinton, S. P34

Cojocneanu-Petric, R. P35

Cominetti, C. P22

Cotter, P. P24

Cuervo, M. P18

Dag, A. P43

De Caterina, R. P29, P30, P31

de la Garza, A.L. P38

de Lorenzo, D. P46

Desmarchelier, C. 09

Deyo, K. 08

Diehl-Jones, W. 011

Domingo, S. 05

Dotan, I. P41

Drbohlav, J. P44

Drori, Y. P45

Dus, I. 014
Eck, P.K. P40

Eventov-Friedman, S. P25

Falach, A. 010, P48

Fedeli, D. 014

Fiorini, D. 014

Fliss-Isakov, N. P41

Frenkel, A.L. 03

Gabbianelli, R. 014, P16

Gadri, Y. 54

Garcia-Calzon, S. 56

Garcia-Lacarte, M. P19

Gatta, V. P29

Go, Y. P15

Godny, L. P41

Golan-Gerstl, R. 013, P26, P32

Goldberg, M.R. P51

Gololobova, Y. 012

Goni, L. P18

González-Muniesa, P. P20, P21

Gophna, U. P41

Granger, E. P34

Gras, M.A. P35, P42

Griess-Fishheimer, S. 01

Grimaldi, K.A. P46

Guelmann-Mizrahi, I. P51

Haile, Z. P25

Hayouka, Z. P50, P52

Holecek, M. P47

Horst, M. P22

Houghton, C. 07

Huang, Z. 04

Huerta, A.E. P17

Jeong, S.W. P15

Jiang, C. 04

Johnson, J. 08

Jones, P.J.H. P40

Joung, H. P53

Juknat, A. 03

Jung, H.-Y. P15

Karani, V. 57

Katz, G. 55

Katz, Y. P51

Kaya, Y. P39

Kerem, Z. P49

Kim, K.T. P15

Kim, M.-Y. P53

Kinra, S. 57
Kisioglu, B. P39

Klein, B. 02

Klumbiene, J. P37

Koren, 0. P28

Kovarik, M. P47

Kuzin, L. 55

Laiglesia, L.M. P21

Lakshmipriya, N. 57

Lasa, A. P20

Le, K. 011

Lebow, M. P45

Lee, D. P15

Lee, H.-S. P53

Lee, I.-K. P15

Lee, S.-J. P15

Leitner, G. 55

Lesauskaite, V. P37

Lesmes, U. P24

Liu, R. 04

Lopez-Pascual, A. P20

Lovegrove, J. 57

Maharshak, N. P41

Mansego, M.L. P19

Marchegiani, F. P16

Marin, D. P35

Marin, D.E. P36, P42

Marta, F.-G. P17

Marti, A. 56, P19

Martinelli, R. P29

Martínez, J.A. 56, P17, P18, P19, P20, P21

Martinez-Fernandez, L. P21

Masci, T. P43

Masisi, K. 011

Massaro, M. P29, P30, P31

Merin, U. 55

Metz, A. P32

Milagro, F.I. P18, P19, P38

Moghadasian, M. 011

Mohan, A.R. 57

Monsonego-Ornan, E. 01

Moraes, C.C. P22

Morell-Azanza, L. 56

Moreno-Aliaga, M.J. 56, P17, P21

Moscovici, A.M. P24

Motiu, M. P36

Napolioni, V. P16

Nasuti, C. 014

Naugolni, L. P51 
Neagoe, I.B. P35

Nehyba, A. P44

Nergiz-Unal, R. P39

Nowicki, M. 09

Oknin, H. 012

Ortíz-López, R. P38

Pasmanik-Chor, M. 03

Peleg, Z. 54

Pellegrino, M. P29, P30, P31

Penn, S. 01

Petkeviciene, J. P37

Pistol, G.C. P35, P36, P42

Polacheck, T. P45

Portillo, M.P. P20

Prieto-Hontoria, P.L. P17

Rachmiel, M. P51

Ramya, K. 57

Rašková, Z.K. P44

Reif, S. 013, P26

Reifen, R. 54, P28, P32

Rendo-Urteaga, T. 56

Riedl, K. P34

Rosenzweig, T. 010, P48, P49

Roubal, P. P44

Rozenberg, K. P49

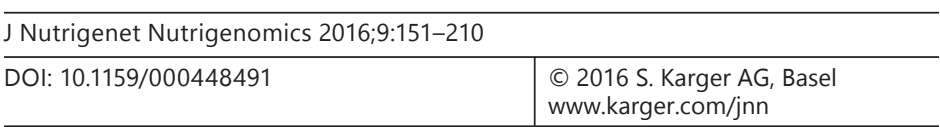

10th Congress of the International Society of Nutrigenetics/Nutrigenomics (ISNN)

Rozenfeld, H. 010, P48

Ryu, H.-G. P15

Safronova, A. P27

Salakova, A. P44

Sampson, S.R. 010, P48, P49

Schroeder, M. P45

Schwartz, S. P34

Scoditti, E. P29, P30, P31

Sedlařík, V. P44

Shafir, S. P43

She, Y. 04

Shemesh, M. 012, P23, P33

Shiff, Y.E. 013, P26, P32

Shilina, N. P27

Shomonov-Wagner, L. 03

Silanikove, N. P25

Sinai, T. P51

Sispera, L. P47

Skalka, N. P49

Skuse, P. P24

Smalinskiene, A. P37

Sorokina, E. P27

Steinberg, D. 012

Stern, T. P52

Storelli, C. P30, P31

Tamir, H. 02
Taranu, I. P35, P36, P42

Teboul, N. 54

Terrero, J. 05

Torres-Villarreal, D. P38

Travinsky, T. 01

Tulchinsky, H. P41

Tzela, C.G. P50

Vasudevan, S. 57

Venkatesan, R. 57

Verdier, J. 04

Vilan, I. 54

Viswanathan, M. 57

Vodenicarovova, M. P47

Vodovotz, Y. P34

Vranceanu, M. P46

Wang, J. 06

Wei, Y. 06

Welch, M. 02

Wiseman, E.M. P28

Yahav, L. P41

Yahav, S. P33

Zarchin, S. P43

Zaretsky, J. 01

Zhang, Y. 04, 06 